\title{
RESEARCH
}

Open Access

\section{Conditioned medium from induced pluripotent stem cell-derived mesenchymal stem cells accelerates cutaneous wound healing through enhanced angiogenesis}

Xiaoting Liang ${ }^{1,2+}$, Fang Lin ${ }^{2,3+}$, Yue Ding ${ }^{4}$, Yuelin Zhang ${ }^{5}$, Mimi Li ${ }^{2}$ Xiaohui Zhou², Qingshu Meng ${ }^{2}$, Xiaoxue Ma², Lu Wei ${ }^{2}$, Huimin Fan ${ }^{2^{*}}$ and Zhongmin Liu ${ }^{1,2,6^{*}}$

\begin{abstract}
Background: Mesenchymal stem cells (MSCs) can improve cutaneous wound healing via the secretion of growth factors. However, the therapeutic efficacy of MSCs varies depending upon their source. Induced pluripotent stem cells are emerging as a promising source of MSCs with the potential to overcome several limitations of adult MSCs. This study compared the effectiveness of conditioned medium of MSCs derived from induced pluripotent stem cells (iMSC-CdM) with that derived from umbilical cord MSCs (UMSC-CdM) in a mouse cutaneous wound healing model. We also investigated the mechanisms of protection.

Methods: The iMSC-CdM or UMSC-CdM were topically applied to mice cutaneous wound model. The recovery rate, scar formation, inflammation and angiogenesis were measured. We compared angiogenesis cytokine expression between iMSC-CdM and UMSC-CdM and their protective effects on human umbilical vein endothelial cells (HUVECs) under $\mathrm{H}_{2} \mathrm{O}_{2}$-induced injury. The effects of iMSC-CdM on energy metabolism, mitochondria fragmentation and apoptosis were measured.

Results: Topical application of iMSC-CdM was superior to the UMSC-CdM in accelerating wound closure and enhancing angiogenesis. Expression levels of angiogenetic cytokines were higher in iMSC-CdM than they were in UMSC-CdM. The iMSC-CdM protected HUVECs from $\mathrm{H}_{2} \mathrm{O}_{2}$ induced injury more effectively than UMSC-CdM did. Administration of iMSC-CdM stimulated HUVEC proliferation, tube formation and energy metabolism via the ERK pathway. Mechanistically, iMSC-CdM inhibited $\mathrm{H}_{2} \mathrm{O}_{2}$-induced mitochondrial fragmentation and apoptosis of HUVECs.

\footnotetext{
* Correspondence: frankfan@tongji.edu.cn; liu.zhongmin@tongji.edu.cn

${ }^{+}$Xiaoting Liang and Fang Lin contributed equally to this work.

${ }^{2}$ Research Center for Translational Medicine, Shanghai East Hospital, School

of Medicine, Tongji University, Shanghai, People's Republic of China

'Institute for Regenerative Medicine, Shanghai East Hospital, School of Life

Sciences and Technology, Tongji University, Shanghai, People's Republic of

China

Full list of author information is available at the end of the article
}

(c) The Author(s). 2021 Open Access This article is licensed under a Creative Commons Attribution 4.0 International License, which permits use, sharing, adaptation, distribution and reproduction in any medium or format, as long as you give appropriate credit to the original author(s) and the source, provide a link to the Creative Commons licence, and indicate if changes were made. The images or other third party material in this article are included in the article's Creative Commons licence, unless indicated otherwise in a credit line to the material. If material is not included in the article's Creative Commons licence and your intended use is not permitted by statutory regulation or exceeds the permitted use, you will need to obtain permission directly from the copyright holder. To view a copy of this licence, visit http://creativecommons.org/licenses/by/4.0/ The Creative Commons Public Domain Dedication waiver (http://creativecommons.org/publicdomain/zero/1.0/) applies to the data made available in this article, unless otherwise stated in a credit line to the data. 
(Continued from previous page)

Conclusion: Collectively, these findings indicate that iMSC-CdM is more effective than uMSC-CdM in treating cutaneous wounds, and in this way, iMSC-CdM may serve as a more constant and sustainable source for cell-free therapeutic approach.

Keywords: Induced pluripotent stem cell-derived mesenchymal stem cells, Conditioned medium, Wound healing, Mitochondria dysfunction

\section{Introduction}

The skin forms a barrier between the inside and the outside of an organism. Injury of the skin may impair its barrier function and expose the inside tissues to pathogens and mechanical damage. In cutaneous wound healing, damaged tissue is repaired through a regenerative process orchestrated by multiple biological pathways [1]. Various therapeutic approaches are available to treat wounds, but current wound-care practices achieve limited effectiveness. Regenerative medicine emerges as an alternative intervention to improve the healing process. Mesenchymal stem cells (MSCs), also known as multipotent mesenchymal stromal cells, are characterized by the capacity for self-renewal and multilineage differentiation. MSCs are one of the most promising tools for regenerative medicine and offer significant therapeutic potential in various diseases, including wound closure. However, more research is needed to determine the ideal sources of therapeutic MSCs and methods for MSC delivery.

The efficacy of MSCs varies depending on their source. Hsieh et al. reported that Wharton jelly MSCs induced better microvasculature formation and cell migration than bone marrow MSCs [2]. In the diabetic mouse wound model, Kim et al. determined that amniotic MSCs significantly enhanced the rate of fibroblast wound closure and re-epithelialization versus adiposederived MSCs [3]. However, there are limitations associated with MSCs derived from adult tissues, including finite cell proliferative capacity, alterations in phenotype and differentiation potential after long-term culture [4]. Therefore, MSCs derived from induced pluripotent stem cells (iMSCs) are emerging as an attractive option, because during the reprogramming process, iMSCs acquire a rejuvenation signature and exhibit improved cellular vitality such as survival, proliferation, and differentiation potential [5]. iMSCs can be expanded up to 120 passages without showing senescent signatures [6] and have therapeutic potential in the regeneration of blood vessels and periodontal, liver, heart, and lung tissues [6-10].

Delivery method is an essential contributing factor in MSC therapy. In many studies of cutaneous wound healing, MSCs were intradermally injected into or around the wound area. Although this method has improved wound healing, poor cell engraftment efficiency limits therapeutic benefits [11]. MSCs exert their therapeutic action mainly by paracrine secretion, such as growth factors, cytokines, chemokines and extracellular microvesicles, into their surroundings. For this reason, cell-free MSC-based approaches have been applied to treat cutaneous wounds. Conditioned medium of Wharton's jellyderived MSCs was more beneficial in the recovery of radiation-induced skin wounds than epidermal growth factor (EGF) [12]. Microencapsulated MSCs attenuated immune cell infiltration, facilitated wound closure and accelerated re-epithelialization [13]. However, the treatment effects of iMSC on cutaneous wound healing have not yet been determined.

The reduced angiogenesis ability is acknowledged as a main contributor to the dysfunctional healing response [14]. Mitochondrial respiration and function control neoangiogenesis in endothelial cells during wound healing $[15,16]$. As highly dynamic organelles, mitochondria can adapt their morphology and function through fusion and fission events in response to environmental change. The fusion process is mediated by mitofusin 1 (MFN1) and mitofusin 2 (MFN2), while fission is accomplished by dynamin-related protein 1 (DRP1). Mutants for mitochondrial fusion and fission proteins fail to close open wounds, indicating the regulation of mitochondrial dynamics is required for wound healing [17]. However, whether iMSC delivered better effects in wound closure through regulating mitochondrial dynamics in endothelial cells remains to be addressed.

In the current study, conditioned medium from iMSCs (iMSC-CdM) and that from umbilical cord MSCs (uMSC-CdM) was topically administered to a mouse cutaneous wound healing model for comparison of their therapeutic efficacies. We revealed that iMSC-CdM was superior to uMSC-CdM in accelerating wound closure with consequent enhanced angiogenesis and regulating the dynamic balance of mitochondria fusion and fission in endothelial cells.

\section{Materials and methods}

Preparation and characterization of iMSCs and uMSCs

Human iMSCs were differentiated from human induced pluripotent stem cell (hiPSC) line IMR90-iPSCs (WiCell Research Institute, Madison, WI, USA) as previously described $[6,18]$. Briefly, hiPSCs were cultured in MSC 
differentiation medium which consists of $10 \%$ fetal bovine serum (FBS), $5 \mathrm{ng} / \mathrm{mL}$ basic fibroblast growth factor (bFGF; AF-100-18B, Peprotech), $5 \mathrm{ng} / \mathrm{mL}$ epidermal growth factor (EGF; AF-100-15, Peprotech) and $55 \mathrm{uM}$ 2-mercaptoethanol (Gibco, 21985023). One week later, differentiating iPSCs were purified by sorting for $\mathrm{CD} 24^{-} \mathrm{CD} 105^{+}$population using a fluorescence-activated cell sorting system. Human umbilical cord MSCs (uMSCs) were purchased commercially (Saliai, Guangzhou, China). MSCs were cultured with $\alpha$-minimum essential medium plus 5\% UltraGRO-Advanced GMP cell culture supplement (Helios, HPCFDCGL50). MSCs were characterized by surface marker profiling (CD34, CD45, CD73, CD90 and CD105) and trilineage differentiation (adipogenesis, osteogenesis and chondrogenesis) as previously described [19]. Cells at passage 4 through passage 8 were used for the following experiments.

\section{Preparation of conditioned medium}

Cells at passage 4 through passage 8 were seeded into $10 \mathrm{~cm}$ culture dishes and allowed to reach $60 \%$ to $70 \%$ confluence. The medium was then changed to $\alpha-$ minimum essential medium $(5 \mathrm{~mL})$, and the cells were cultured for another $24 \mathrm{~h}$. Next, the conditioned medium was collected, centrifuged to remove the debris, filtered, and stored at $-80^{\circ} \mathrm{C}$.

\section{In vivo wound generation and macroscopic examination}

Male Balb/C mice, 6 to 8 weeks old, were purchased from Vital River (Beijing, China). Mice were randomly assigned to iMSC-CdM, uMSC-CdM, or vehicle control groups (10 in each group). All mice were anesthetized by intraperitoneal injection of $50 \mathrm{mg} / \mathrm{kg}$ pentobarbital before surgery. A 10-mm-diameter, full-thickness excisional wound was created under sterile surgical conditions. The wounds were covered with a sterile cotton pad followed by topical administration of uMSC-CdM or iMSC-CdM $(100 \mu \mathrm{L})$. An equivalent volume of $\alpha-$ minimum essential medium was administered in the control group. Thirty minutes later, the cotton pad was removed from the wounds. The treatment mentioned above was conducted daily. The epithelial gap was measured every day. The recovery rate was expressed as a percentage of the original wound size with the following formula: (1-remaining wound size/original wound size) $\times 100 \%$. Mice were sacrificed at 9 days after surgery.

Immunohistochemical and immunofluorescence analyses The collected specimens were fixed in $4 \%$ paraformaldehyde solution, dehydrated with a series of graded ethanol, and embedded in paraffin. Sections were stained with Masson trichrome and photographed under an optical microscope. Immunofluorescence staining for
CD31 (Abcam, ab76533), vimentin (Cell Signaling, 5741), and cytokeratin (Abcam, ab9377) was performed to assess the extent of newly formed microvessels, fibroblasts, and keratinocytes, respectively. Six random fields per section near wound edges were counted with ImagePro Plus 6 software.

\section{Cytokine profiling}

The skin specimens were lysed with lysis buffer (Cell Signaling, 9803). Proteins were quantified by BCA Protein Assay Kit (Thermo Fisher, 23327). The expression levels of interleukin-6 (IL6), monocyte chemoattractant protein-1 (MCP1), interferon- $\gamma$ (IFN $\gamma)$, interleukin-10 (IL10), interleukin-12p70 (IL12p70), and tumor necrosis factor-alpha (TNF $\alpha)$ were measured with a bead-based analyte detection system (BD Biosciences, 552364) according to the manufacturer's instructions. The expression of EGF and vascular endothelial growth factor A (VEGFA) was determined by enzyme-linked immunosorbent assay (ELISA) according to the manufacturer's instructions (Multiscience, EK283 \& EK293).

\section{Primary skin fibroblast isolation}

Primary skin fibroblasts were isolated as previously described [20]. Briefly, skin tissues were obtained from 1 to 3 days old mice. The skin tissues were cut into approximately $1 \mathrm{~mm}$ pieces and digested with $2 \mathrm{mg} / \mathrm{mL}$ type I collagenase (Sigma-Aldrich, C0130) for $2 \mathrm{~h}$. After washing, the precipitate was resuspended in the culture medium (DMEM/F12 supplemented with 15\%FBS) and transferred to a $10-\mathrm{cm}$ dish. The fibroblasts started to exit tissue fragments within 1 week.

\section{Transwell assay}

To determine whether uMSC-CdM/iMSC-CdM affected cell mobility, $2 \times 10^{4}$ skin fibroblasts were seeded on the upper chamber of the transwell apparatus with a pore size of $8 \mu \mathrm{m}$ (Corning, 3422). In the lower chamber, uMSC-CdM and iMSC-CdM supplemented with $2 \%$ FBS were added as attractants, and basal medium supplemented with $2 \%$ FBS was set as the control group. Six hours later, the cells were fixed using $4 \%$ paraformaldehyde for $30 \mathrm{~min}$. Non-migrated cells were scraped off the upper surface of the membrane with a cotton swab. Migrated cells that remained on the lower surface were stained with 2-(4-Amidinophenyl)-6-indolecarbamidine dihydrochloride (DAPI). There were triplicate wells with 6 random sights for each well for each group.

\section{Quantitative reverse transcription polymerase chain reaction}

Total RNA was extracted from skin specimens with RNeasy Mini Kit (Qiagen, 74124). cDNA was synthesized from $500 \mathrm{ng}$ of total RNA with a RevertAid First 
Strand cDNA Synthesis Kit (Takara, RR0036A). Then, quantitative reverse transcription polymerase chain reaction analysis was performed with Fast SYBR ${ }^{\mathrm{ma}}$ Green Master Mix (4385617) in an ABI QuantStudio 6 Flex System. The relative standard curve method $\left(2^{-\triangle \Delta C T}\right)$ was used to determine the relative mRNA expression, with glyceraldehyde 3-phosphate dehydrogenase (GAPD $\mathrm{H})$ as the reference gene. The polymerase chain reaction primers used in this study are listed in Table 1. Mitochondrial energy metabolism PCR array (Qiagen, PAHS008Y) was performed to detect the expression profile of 84 genes associated with mitochondrial respiration, including genes encoding components of the electron transport chain and oxidative phosphorylation complexes. The median cycle threshold value $(\mathrm{CT})$ was uploaded onto the manufacturer's web portal (https:// geneglobe.qiagen.com/cn/analyze), and the fold change of each gene expression was calculated using the provided software according to the manufacturer's instruction.

\section{Cell apoptosis, reactive oxygen species, MitoSOX ${ }^{\mathrm{TM}}$, and mitochondrial permeability transition pore staining} Cells at $60 \%$ to $70 \%$ confluence were treated with $800 \mu \mathrm{M} \mathrm{H} \mathrm{H}_{2} \mathrm{O}_{2}$ for $24 \mathrm{~h}$. A total of $1 \%$ FBS was added to avoid severe cell injury, which is optimal to determine the protective effects of the intervention. The cell

Table 1 Primer sequence

\begin{tabular}{ll}
\hline Gene & Sequence \\
\hline Mouse ANG-2 forward & CTCTGTCTCAGGATGACTCCAG \\
Mouse ANG-2 reverse & AGGTGTTGACATCTTGCAGAAAG \\
Mouse EGF forward & ACTGGTGTGACACCAAGAGGTC \\
Mouse PIGF forward & CCACAGGTGATCCTCAAACACG \\
Mouse PIGF reverse & AGTTCACAGGAGCGTGGCTTG \\
Mouse VEGFA forward & GATCCAGAGTGGCGAGATAACC \\
Mouse VEGFA reverse & CTGCTGTAACGATGAAGCCCTG \\
Mouse GAPDH forward & GCTGTAGGAAGCTCATCTCTCC \\
Mouse GAPDH reverse & CATCACTGCCACCCAGAAGACTG \\
Human EGF forward & ATGCCAGTGAGCTTCCCGTTCAG \\
Human EGF reverse & TGCGATGCCAAGCAGTCTGTGA \\
Human FGF2 forward & GCATAGCCCAATCTGAGAACCAC \\
Human FGF2 reverse & AGCGGCTGTACTGCAAAACGG \\
Human HGF forward & CCTITGATAGACACAACTCCTCTC \\
Human HGF reverse & GAGAGTTGGGTCTTACTGCACG \\
Human VEGFA forward & CTCATCTCCTCTTCCGTGGACA \\
Human VEGFA reverse & TTGCCTTGCTGCTCTACCTCCA \\
Human GAPDH forward & GATGGCAGTAGCTGCGCTGATA \\
Human GAPDH reverse & GTCTCCTCTGACTTCAACAGCG \\
\hline
\end{tabular}

apoptosis was determined with an AnnexinV-APC/PI apoptosis detection kit (Sony Biotechnology, 3804660) according to the manufacturer's instructions. Cell reactive oxygen species (ROS) were determined by the Total Reactive Oxygen Species Assay Kit (Thermo Fisher, 885930-74). Mitochondrial superoxide was stained with MitoSOX $^{\mathrm{si}}$ red indicator (Thermo Fisher, M36008). Mitochondrial permeability transition pore (mPTP) opening was determined with the MitoProbe ${ }^{\text {tw }}$ Transition Pore Assay Kit (Thermo Fisher, M34153).

\section{Proliferation assay}

The effects of MSC-CdM on HUVECs and skin fibroblasts proliferation were determined with the Cell Counting Kit-8 assay (Dojindo, CK04). Briefly, $1 \times 10^{3}$ cells per well (4 replicates per group) were seeded into 96-well plates and cultured in the medium as indicated. At indicated time points, Cell Counting Kit-8 solution $(10 \mu \mathrm{L})$ and $100 \mu \mathrm{L}$ of fresh culture medium were added to each well and incubated at $37^{\circ} \mathrm{C}$ for $1 \mathrm{~h}$. The absorbance was observed at $450 \mathrm{~nm}$ with a microplate reader. The survival/proliferation of cells was calculated as the absorbance of the test wells minus the optical density of the blank wells.

\section{Tube formation assay}

Growth Factor Reduced Matrigel (BD Biosciences, 356231) was plated in 96-well plates and incubated at $37^{\circ} \mathrm{C}$ for $30 \mathrm{~min}$. Then, human umbilical vein endothelial cells (HUVECs) were seeded on polymerized Matrigel at $1 \times 10^{4}$ per well ( 4 replicates per group), and the medium was added as indicated. A total of $10 \mathrm{uM}$ U0126 (MCE, HY-12031) was added in the U0126treated groups. After incubation at $37^{\circ} \mathrm{C}$ for $4 \mathrm{~h}$, tube formation was recorded with an inverted microscope. The total branching points and total tube length were measured with Image-Pro Plus 6 software.

\section{Protein array}

Angiogenic protein concentration in $\mathrm{uMSC}-\mathrm{CdM}$ and iMSC-CdM was determined with an antibody array (Raybiotec, QAH-ANG-1). This multiplexed sandwich ELISA-based quantitative array platform detects 10 proteins. Pooled conditioned medium from uMSC $(n=3)$ and iMSC $(n=3)$ was used for the experiment according to the manufacturer's instructions. The signals were captured by a laser scanner InnoScan 300 equipped with a Cy3 wavelength and analyzed by the microarray analysis software.

\section{ATP concentration assessments}

A total of $20 \times 10^{4}$ cells/per well were seeded into 6 well plates, and $2 \mathrm{~mL}$ DMEM, uMSC-CdM, or iMSC-CdM was added in indicated groups, respectively. The 
inhibitors including $10 \mathrm{uM} \mathrm{U0126}$ (MCE, HY-12031), $20 \mathrm{uM}$ SB203580 (MCE, HY-10256), and $10 \mathrm{uM}$ LY294002 (MCE, HY-10108) were added in indicated groups, respectively. Twenty-four hours later, cells were harvested for in vitro adenosine triphosphate (ATP) concentration analysis. ATP concentrations were determined with an ATP assay kit (Beyotime, S0027) according to the manufacturer's instructions.

\section{Oxygen consumption rate measurement}

A Seahorse XFp Analyzer (Agilent Technologies, RRID: SCR_013575) was applied to evaluate the mitochondrial function as previously described [21]. Briefly, $1.5 \times 10^{4}$ HUVECs were seeded in each well of an XF cell culture microplate with $180 \mu \mathrm{L}$ culture medium. U0126 was added at a concentration of $10 \mathrm{uM}$ in the iMSC-CdM + U0126 group. Cells were incubated overnight at $37^{\circ} \mathrm{C}$ in $5 \% \mathrm{CO}_{2}$, and then the culture medium was replaced with $180 \mu \mathrm{L}$ of XF Medium. The bioenergetic profile was characterized with the following 4 parameters: (i) basal respiration in assay medium with pyruvate $(0.6 \mathrm{mM}), \mathrm{L}-$ glutamine $(6.98 \mathrm{mM})$, and D-glucose $(5.78 \mathrm{mM})$; (ii) post inhibition ATP synthase activity by $1.5 \mu \mathrm{M}$ oligomycin as well as respiration-driving proton leak and ATP synthesis turnover; (iii) maximal mitochondrial respiratory capacity following treatment with the uncoupling agent $0.5 \mu \mathrm{M} \quad$ FCCP (carbonyl cyanide ptrifluoromethoxyphenylhydrazone); and (iv) nonmitochondrial respiration measured posttreatment with $2 \mu \mathrm{M}$ complex I inhibitor rotenone and complex III inhibitor antimycin A.

\section{siRNA transfection}

MFN1 siRNA was used to transfect HUVECs using Lipofectamine RNAiMAX (Thermo, 13778-075) according to the manufacturer's instructions. HUVECs at $70-80 \%$ confluence were transfected and incubated for $48 \mathrm{~h}$. The transfection efficiency was determined by Western blot analysis.

\section{MitoTracker staining}

The morphology of mitochondria was examined by MitoTracker staining (Cell Signaling, 9074) as previously described [22]. The percentage of mitochondrial fragmentation was calculated by comparing the number of cells with fragmented mitochondria to the total number of cells.

\section{Western blotting}

Protein extracts were separated by sodium dodecyl sulfate-polyacrylamide gel electrophoresis and transferred to polyvinylidene fluoride membranes as previously described [23]. The membranes were incubated with the following antibodies: anti-VEGFA (Proteintech,
66828-1-1 g); anti-pERK1/2 (Cell Signaling, 4370), antiERK1/2 (Cell Signaling, 4695), anti-MFN1 (Proteintech, 13798-1-AP), anti-MFN2 (Proteintech, 12186-1-AP), anti-pDRP1 ser616 (Cell Signaling, 3455), anti-DRP1 (Proteintech, 12957-1-AP), and anti- $\beta$-actin (Cell Signaling, 3700), at $4{ }^{\circ} \mathrm{C}$ overnight. After washing, the membrane was incubated with the horseradish peroxidaseconjugated secondary antibodies at $37^{\circ} \mathrm{C}$ for $1 \mathrm{~h}$. The immunoreactive bands were visualized with enhanced chemiluminescence reagent (Thermo Fisher, 32109) and imaged with the ChemiDoc XRS Plus luminescent image analyzer (Bio-Rad). Densitometric quantification of band intensity from 4 independent experiments was conducted with Image-Pro Plus 6.0 software.

\section{Statistical analysis}

All experiments were performed with at least 3 replicates per group, and the in vitro experiments were repeated at least 3 times. Data are representative of these experiments and are shown as means plus or minus standard error of the mean. The means of multiple groups were compared with the one-way analysis of variance (ANOVA). Statistical analyses were conducted with GraphPad Prism software, and $P<.05$ was considered statistically significant.

\section{Results}

Characterization of UMSC and iMSC and preparation of uMSC-CdM/iMSC-CdM

MSCs at passage 4 were characterized by surface marker profiling and differentiation capacity. Both uMSCs and iMSCs displayed positive expression of MSC markers CD105, CD90 and CD73, and negative expression of CD34 and CD45 (Fig. 1a). The uMSCs and iMSCs showed a spindle shape and multiple differentiation potential toward adipogenesis, chondrogenesis and osteogenesis (Fig. 1b). A schematic diagram of uMSC-CdM and iMSC-CdM preparation is shown in Fig. 1c.

\section{iMSC-CdM treatment is more effective than UMSC-CdM in accelerating wound closure}

After wound induction, the wounds were treated daily by topical administration of medium to a sterile cotton pad covering the wound and surrounding area (Fig. 2a, b). Mice in the control group lost weight in the first 4 days after wound induction, while mice in the conditioned medium groups maintained their body weight (Supplementary Figure 1). Conditioned medium significantly accelerated wound closure versus the control group (Fig. 2c, d). Notably, the iMSC-CdM treated group showed more prominent therapeutic effects than the uMSC-CdM group (Fig. 2c, d). 


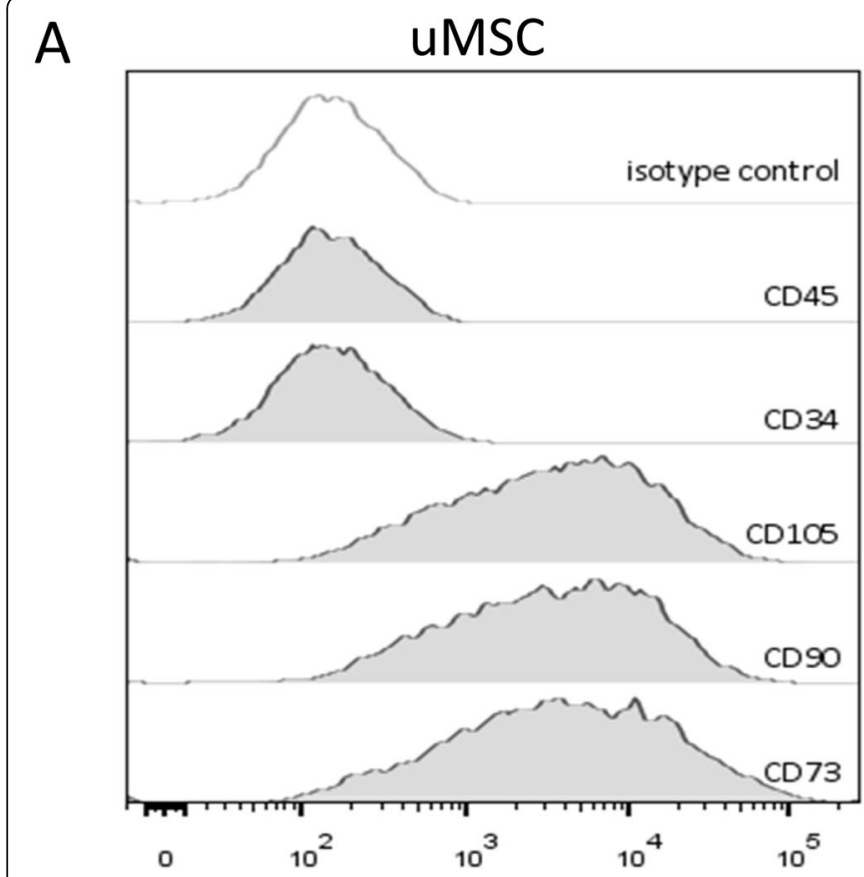

B

\section{$\sum_{J}^{U}$}
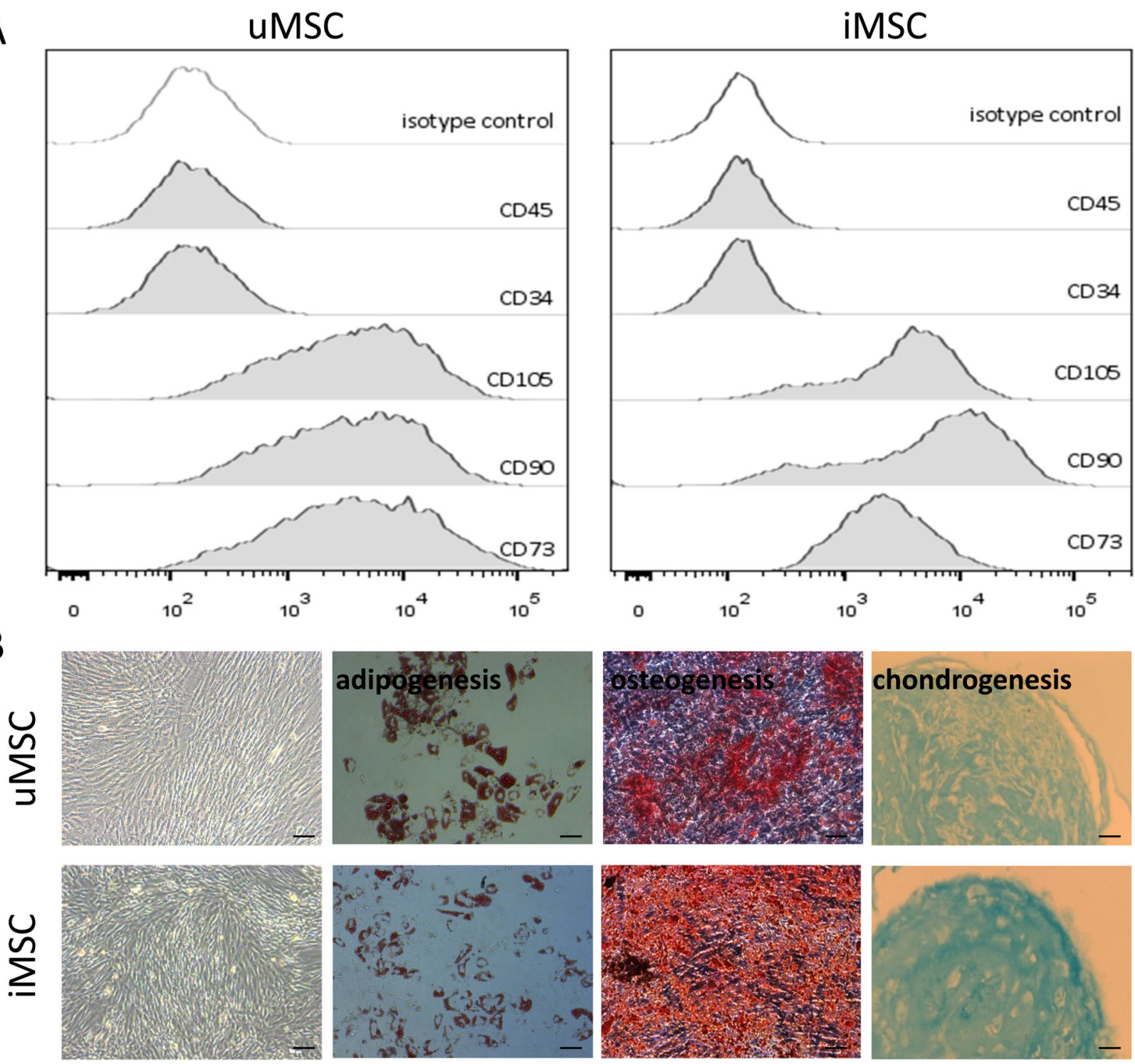

C

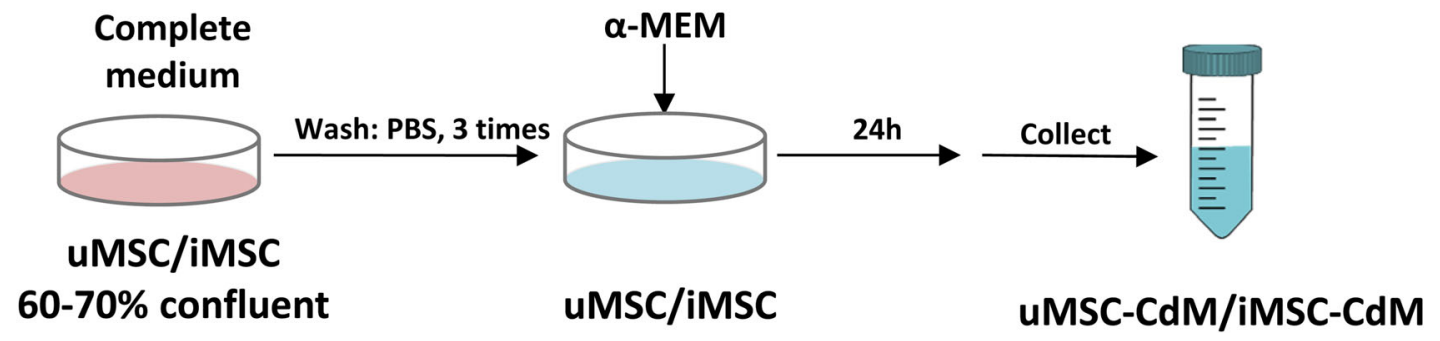

Fig. 1 Characterization of mesenchymal stem cells derived from umbilical cord mesenchymal stem cells (uMSCs) and mesenchymal stem cells derived from induced pluripotent stem cells (iMSCs). a Surface marker profiling was determined by flow cytometry. b Morphology and trilineage differentiation of uMSCS and iMSCs. Scale bar $=100 \mu \mathrm{m}$. c Schematic diagram for conditioned medium collection 


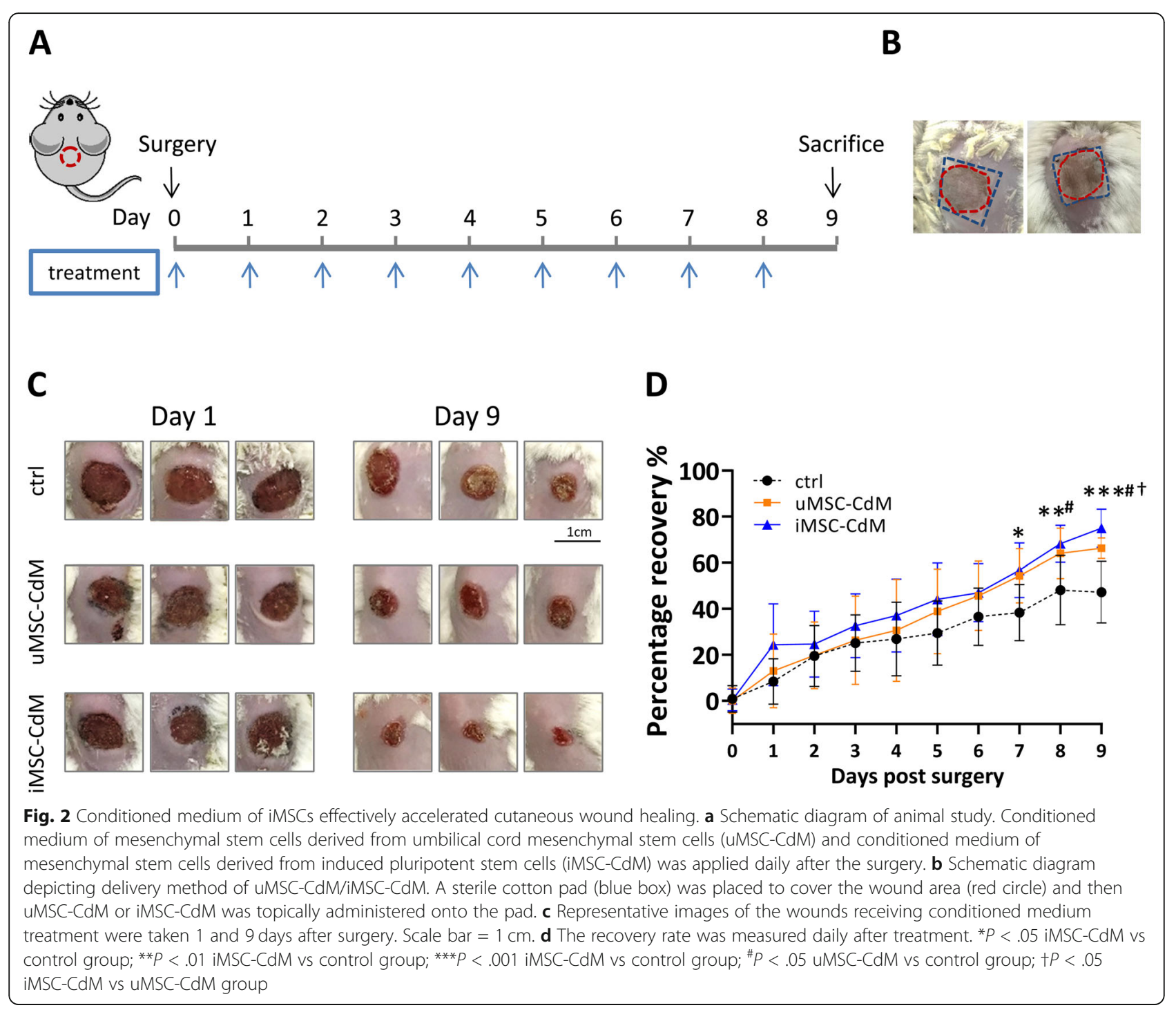

iMSC-CdM treatment inhibits inflammation and increases neovascularization of wounded tissue

Trichrome staining of wound sections revealed that uMSC-CdM- and iMSC-CdM-treated mice had similar granulation tissue at 9 days after wound induction. By contrast, the control group lacked blue-stained collagen fibers (Fig. 3a). The conditioned medium treatment inhibited inflammatory cytokine secretion, including IL6, MCP1 and TNF $\alpha$, versus the control group, and iMSC$\mathrm{CdM}$ was more effective than uMSC-CdM (Fig. 3b). The other three cytokines, including IFN $\gamma$, IL10 and IL12p70 showed a low expression out of the measuring range limit in all three groups (data not shown). MSCs can activate the angiogenesis, proliferation, migration, and differentiation of the main cell types involved in skin regeneration, including endothelial cells, fibroblasts, and keratinocytes [24]. We isolated skin fibroblasts and determined whether uMSC-CdM/iMSC-CdM affects the proliferation and migration of fibroblasts by CCK8 assay and transwell assay, respectively. As shown in Supplementary Figure 2A, uMSC-CdM and iMSC-CdM increased proliferation of skin fibroblasts at day 3 , and there was no difference between the uMSC-CdM group and iMSC-CdM group. The uMSC-CdM and iMSC$\mathrm{CdM}$ did not affect the mobility of skin fibroblasts (Supplementary Figure 2B). Immunostaining showed an enhanced expression of fibroblast marker vimentin in the conditioned medium treated groups compared to the control group, although no significant difference was detected between the uMSC-CdM group and iMSC-CdM group (Supplementary Figure 2C). To evaluate the effects of uMSC-CdM/iMSC-CdM on keratinocytes, immunostaining against keratinocyte marker cytokeratin was performed. The data showed an enhanced cytokeratin expression in the conditioned medium treated groups compared to the control group. Still, there was no 
A
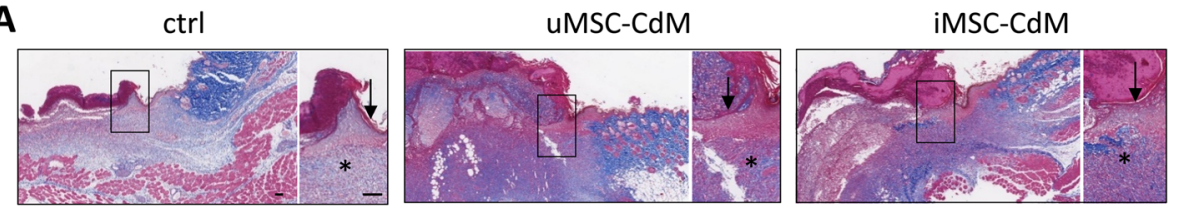

B
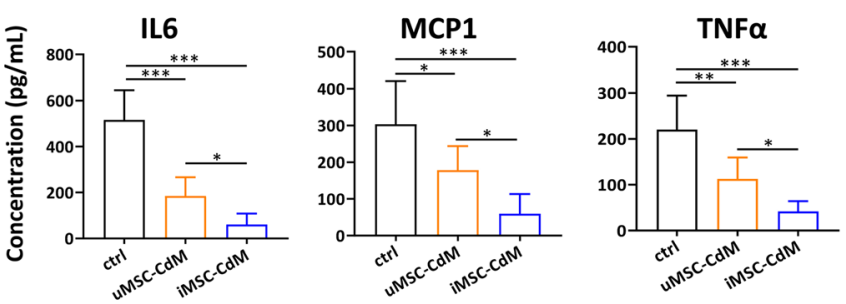

C
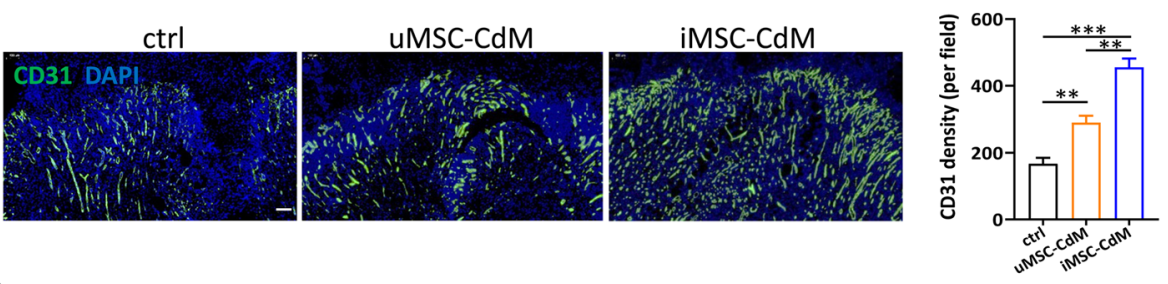

D

ANG-2
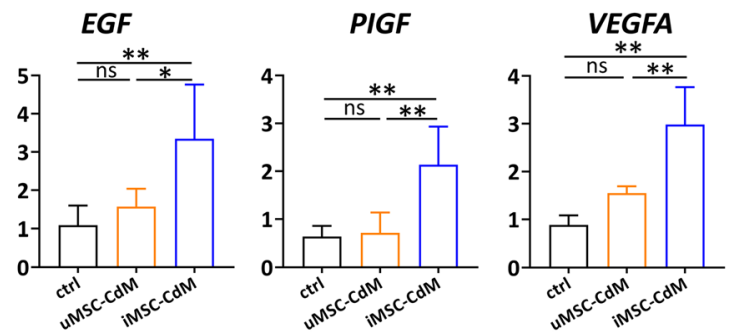

E

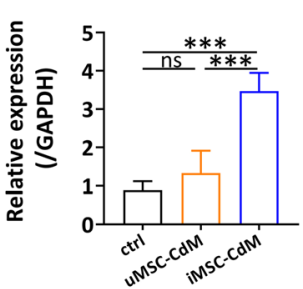

VEGFA
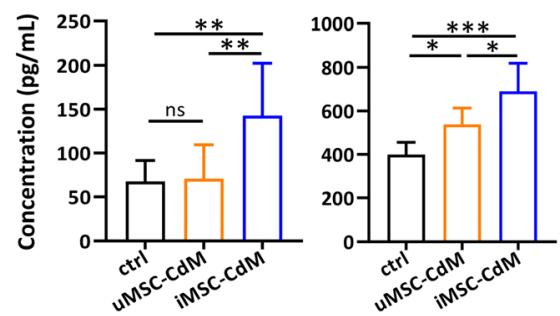

Fig. 3 Treatment with iMSC-CdM inhibited inflammation and enhanced angiogenesis. a Trichrome staining revealed that the wounds treated with UMSC-CdM and iMSC-CdM had similar granulation tissue at the peri-wound area (arrows indicate keratinocytes; stars indicate granulation tissue). Scale bar $=100 \mu \mathrm{m}$. b Inflammatory cytokine expression was determined by a bead-based immunoassay. c Representative fluorescence images and quantification of CD31 positive blood vessels. Scale bar $=100 \mu \mathrm{m}$. $\mathbf{d}$ Expression of pro-angiogenesis factors at the peri-wound area determined by quantitative polymerase chain reaction. e EGF and VEGFA protein expression determined by Elisa assay. ns, non-significance, ${ }^{*} P<$ $.05,{ }^{* *} P<.01,{ }^{* *} P<.001$

significant difference between the uMSC-CdM treated group and iMSC-CdM treated group (Supplementary Figure 2D). Collectively, these data suggested uMSCCdM and iMSC-CdM have positive effects on keratinocytes and fibroblasts. However, there was no significant difference between the uMSC-CdM and iMSC-CdM groups. Angiogenesis is an essential component of wound repair, as vessels support cells at the wound site with nutrition and oxygen. We then determined the capillary density of the peri-wound area by immunostaining with anti-CD31 (a marker of endothelial cells). Capillary density was enhanced in the conditioned medium treated groups versus the control group, with more significant enhancement in the iMSC-CdM group than in the uMSC-CdM group (Fig. 3c). The expression level of angiogenesis factors, including angiopoietin 2 (ANG-2), EGF, placenta growth factor (PIGF) and VEGFA, at the peri-wound area, were further measured by quantitative polymerase chain reaction analysis. While the uMSC-CdM treatment showed marginally 
higher expressions of ANG-2, EGF, PIGF and VEGFA versus the control (statistically not significant), the iMSC-CdM treatment increased the expression of the cytokines as mentioned above (Fig. 3d). Further, we measured the protein expression of EGF and VEGFA by ELISA assay. There was no significant difference in EGF protein expression between the control and uMSC-CdM treated group (Fig. 3e). Nevertheless, the VEGFA expression was significantly higher in the conditioned medium treated groups compared to the control group. Notably, the iMSC-CdM-treated group exhibited a higher EGF and VEGF expression compared to the control and uMSC-CdM treated groups, respectively (Fig. 3e). Taken together, these data showed that external application of iMSC-CdM inhibited inflammation and increased neovascularization of wounded tissue.

\section{The cytoprotective effects and angiogenic potential of iMSC-CdM on HUVECs}

Enhanced angiogenesis in vivo suggested a protective function of conditioned medium on endothelial cells. We thus determined the cytoprotective effects of conditioned medium on HUVECs under $\mathrm{H}_{2} \mathrm{O}_{2}$-induced stress. $\mathrm{H}_{2} \mathrm{O}_{2}$ induced HUVEC apoptosis in controls, and conditioned medium treatment significantly reduced apoptosis (Fig. 4a). The iMSC-CdM group showed a more protective effect than the uMSC-CdM group (Fig. 4a). Similarly, conditioned medium significantly reduced levels of cellular ROS induced by $\mathrm{H}_{2} \mathrm{O}_{2}$ treatment, and iMSC-CdM was more effective in reducing cellular ROS than uMSCCdM (Fig. 4b). We further determined the levels of mitochondrial ROS and showed that iMSC-CdM and uMSC-CdM were equivalent in inhibiting mitochondrial ROS (Fig. 4c). The mitochondrial permeability transition pore (mPTP) opening was analyzed as the capacity of mitochondria to retain the dye calcein-AM in presence of $200 \mu \mathrm{M} \mathrm{CaCl}_{2}$. The data showed that $\mathrm{H}_{2} \mathrm{O}_{2}$ markedly induced $\mathrm{MPTP}$ opening, as evidenced by reduced calcein-AM intensity (Fig. 4d). The effect of $\mathrm{H}_{2} \mathrm{O}_{2}$-induced mPTP opening was prevented by the conditioned medium treatment, and iMSC-CdM provided more protection than uMSC-CdM, as evidenced by increased calcein-AM intensity (Fig. 4d). Under normal culture conditions, conditioned medium treatment significantly increased proliferation rate in HUVECs, and at day 4, iMSC-CdM had a more substantial effect than uMSCCdM (Fig. 4e). The angiogenic capacity of the conditioned medium was analyzed by tube formation assay, which showed that iMSC-CdM treatment increased endothelial network formation more than uMSC-CdM treatment (Fig. 4f). With equivalent cell numbers, the protein concentration of iMSC-CdM was higher than uMSC-CdM, and the levels of angiogenic factor angiogenin, VEGFA, PDGF-BB, HGF and FGF2 were higher in iMSC-CdM than they were in uMSC-CdM (Fig. 4g). Collectively, these data suggested that iMSC-CdM presented more significant cytoprotective effects and angiogenic potential than uMSC-CdM.

\section{iMSC-CdM enhanced energy metabolism and angiogenesis via activating ERK signaling pathway}

To evaluate the molecular mechanisms by which conditioned medium facilitated angiogenesis, HUVECs were treated with pathway inhibitors U0126 (ERK inhibitor), SB203580 (p38 MAPK inhibitor), and LY294002 (PI3K/ Akt inhibitor), and ATP concentrations were measured. Among the 3 inhibitors, U0126 showed the most prominent inhibition in ATP content in both the uMSC-CdM and iMSC-CdM groups, suggesting that the ERK signaling pathway might play a predominant role in regulating conditioned medium-mediated cytoprotection (Fig. 5a). The effects of conditioned medium on the energy metabolism of HUVECs were then examined. The oxygen consumption rate (OCR) of HUVECs was determined with an extracellular flux analyzer. Conditioned medium treatment significantly increased the basal respiratory capacity and ATP production of HUVECs (Fig. 5b). Supplementation of U0126 significantly decreased basal respiratory capacity and ATP production mediated by conditioned medium treatment (Fig. 5b). Similarly, iMSC-CdM treatment enhanced tube formation (Fig. $5 \mathrm{c}$ ), increased protein expression of ERK, p-ERK and VEGFA (Fig. 5d), and induced angiogenic factor expression including EGF, FGF2, HGF and VEGFA (Fig. 5e) more than uMSC treatment did. U0126 supplementation partially neutralized the effects of iMSC-CdM on tube formation (Fig. 5c), protein expression (Fig. 5d), and angiogenic cytokine expression (Fig. 5e). Collectively, these data suggested that iMSC-CdM enhanced energy metabolism and angiogenesis by activating the ERK signaling pathway.

\section{iMSC-CdM inhibited $\mathrm{H}_{2} \mathrm{O}_{2}$-induced mitochondrial fragmentation and apoptosis of HUVECs}

Mitochondria are the power generators of the cell, converting oxygen and nutrients into ATP. High ROS levels may release a ROS burst leading to the destruction of mitochondria or even the cell. We have shown iMSCCdM protected HUVECs from $\mathrm{H}_{2} \mathrm{O}_{2}$ induced-apoptosis and ROS production (Fig. 4), and iMSC-CdM increased the basal respiratory capacity and ATP production of HUVECs (Fig. 5a, b). We then determined whether iMSC-CdM regulated $\mathrm{H}_{2} \mathrm{O}_{2}$-induced mitochondrial fragmentation in HUVECs. We showed the iMSC-CdM treatment significantly reduced $\mathrm{H}_{2} \mathrm{O}_{2}$-induced mitochondrial fragmentation in HUVECs (Fig. 6a). Western blotting demonstrated downregulation of p-DRP1 ser616 and upregulation of MFN1 in the iMSC-CdM treated 
A

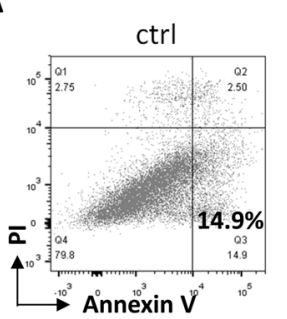

B

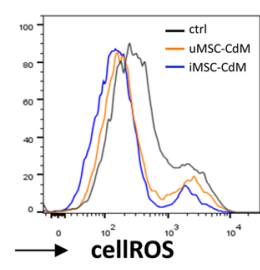

D
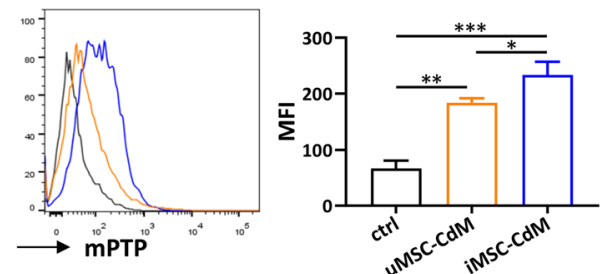

$\mathbf{F}$

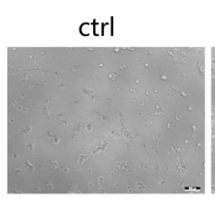

uMSC-CdM
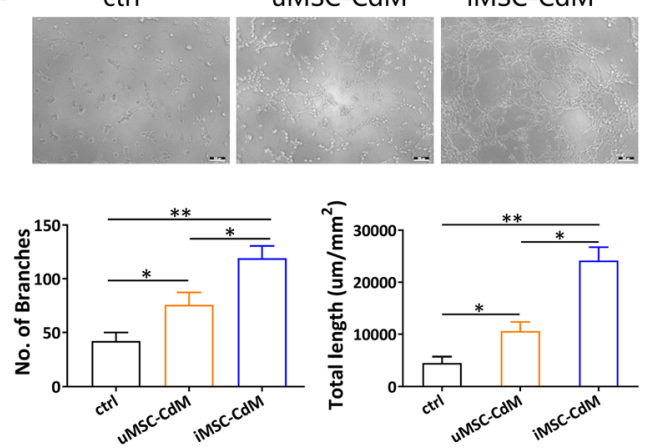

C

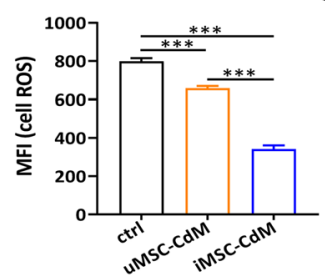

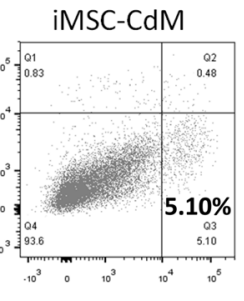
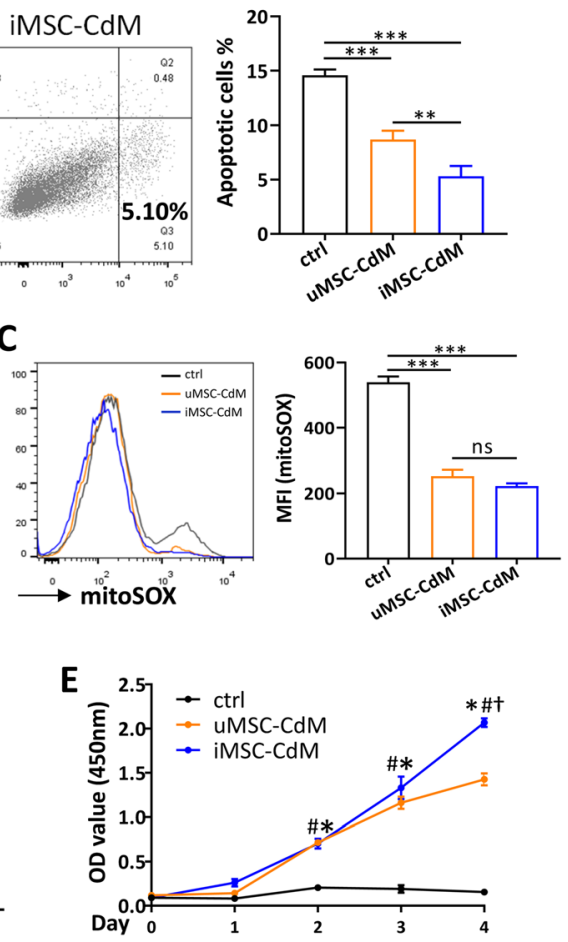

G
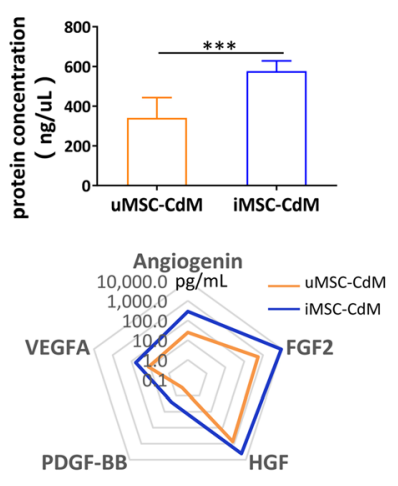

Fig. 4 The cytoprotective effects of iMSC-CdM on $\mathrm{H}_{2} \mathrm{O}_{2}$ treated HUVECs. After $\mathrm{H}_{2} \mathrm{O}_{2}$ treatment, a apoptosis, b cell reactive oxygen species, $\mathbf{c}$ mitochondrial reactive oxygen species, and $\mathbf{d}$ mitochondrial permeability transition pore (mPTP) opening were determined by flow cytometry. MFI, mean fluorescent intensity. ns, non-significance, ${ }^{*} P<.05$, ${ }^{* *} P<.01,{ }^{* * *} P<.001$. e Cell proliferation was determined by Cell Counting Kit-8 assay. ${ }^{*} P<.05$ iMSC-CdM vs control group; $\# P<.05$ UMSC-CdM vs control group; $+P<.05$ iMSC-CdM vs uMSC-CdM group. f Representative light photomicrographs and quantitative analysis of HUVEC tube formation assay after UMSC-CdM/iMSC-CdM treatment. Scale bar $=100 \mu \mathrm{m}$. ${ }^{*} P<.05$, ${ }^{* *} P<.01 . \mathbf{g}$ Protein concentration and expression levels of angiogenic factors in UMSC-CdM and iMSC-CdM. ${ }^{* *} P<.001$

group compared to the non-treated group (Fig. 6b). There was no difference in MFN2 expression between the iMSC-CdM treated and non-treated groups (Fig. 6b). Moreover, the iMSC-CdM treatment decreased $\mathrm{H}_{2} \mathrm{O}_{2}$ induced apoptosis compared to the non-treated group (Fig. 6c). We then determined whether iMSC-CdM exerted protective effects through regulating mitochondria dynamics. As MFN1 was upregulated in the iMSC$\mathrm{CdM}$ treated group compared to the non-treated group (Fig. 6b), we applied MFN1-siRNA to suppress its expression in the iMSC-CdM treated group, and then mitochondria fragmentation and apoptosis were examined. The elevation of MFN1 expression induced by iMSC-CdM treatment under $\mathrm{H}_{2} \mathrm{O}_{2}$ stimulation was inhibited by MFN1 siRNA (Fig. 6b). Notably, the MFN1 siRNA treatment partially neutralized the effects of iMSC-CdM on mitochondria fragmentation (Fig. 6a) and apoptosis (Fig. 6c). A mitochondrial energy metabolism PCR array was applied to determine the expression profile of 84 key genes related to mitochondria biogenesis 

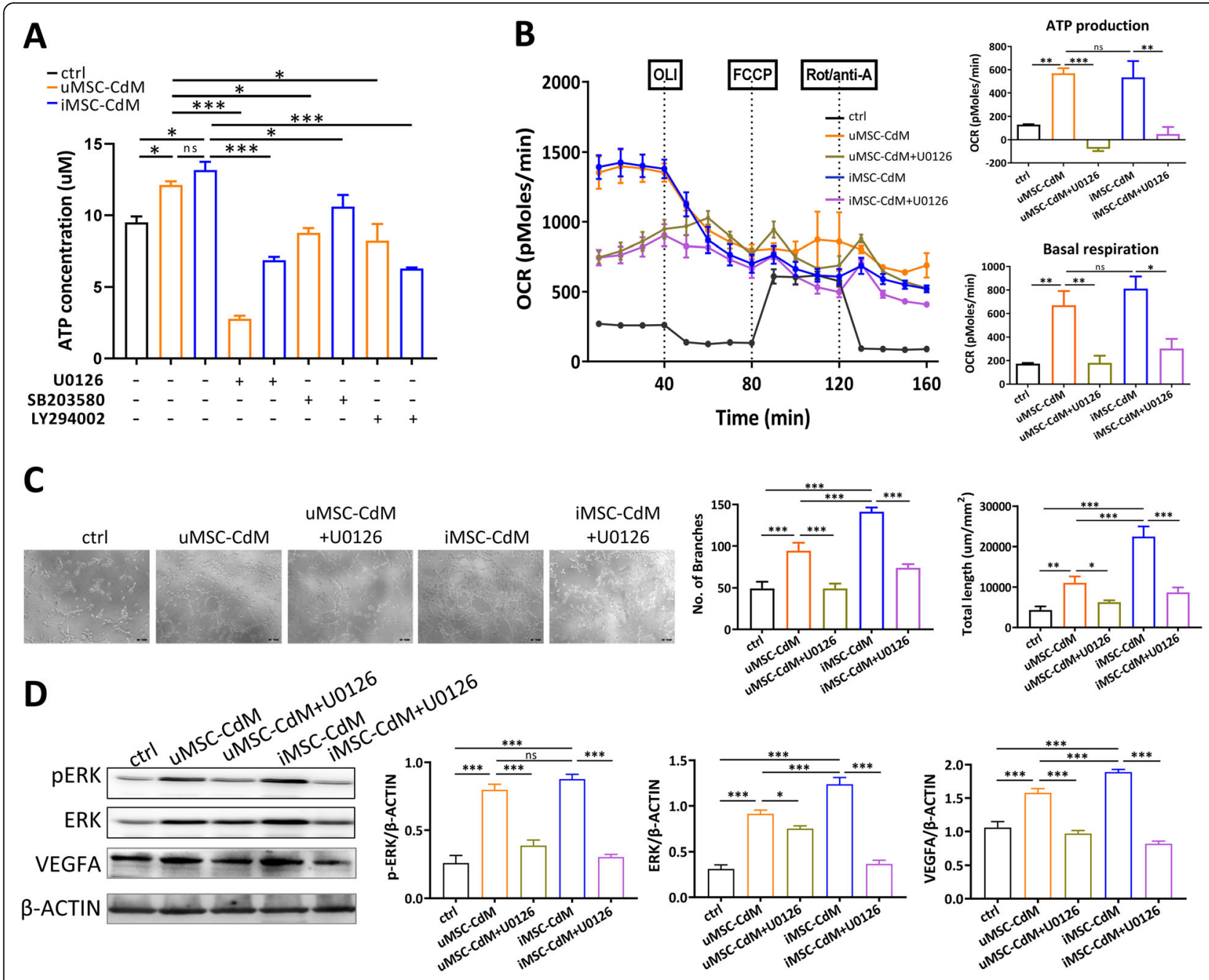

$\mathbf{E}$
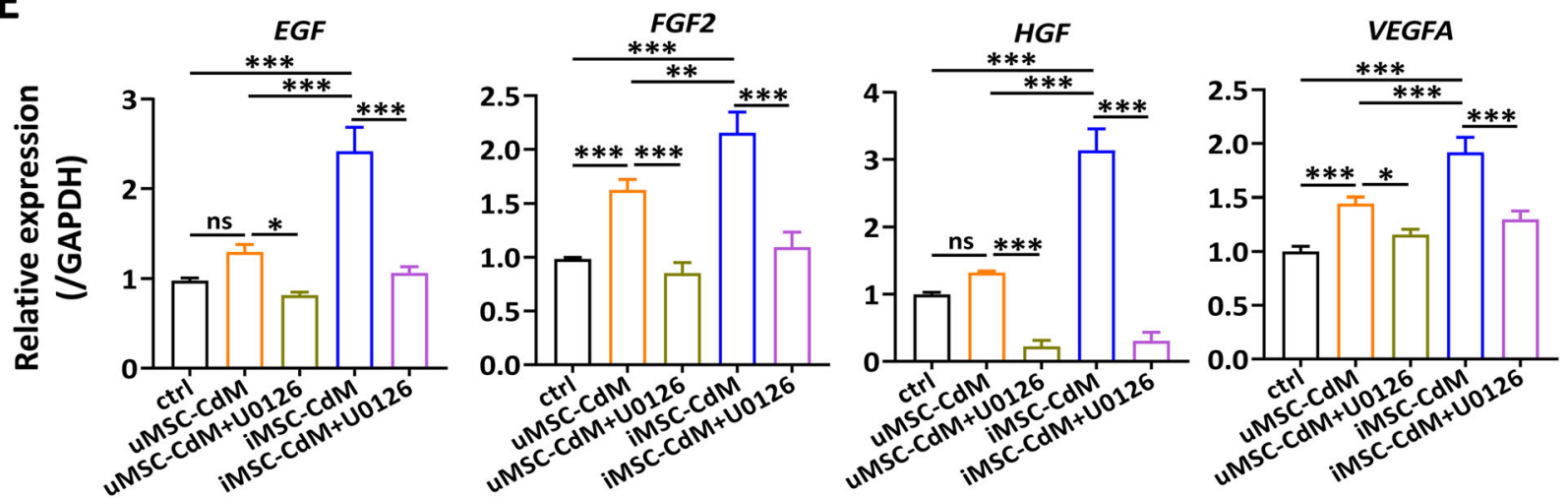

Fig. 5 iMSC-CdM enhanced energy metabolism and angiogenesis via the ERK pathway. a ATP concentrations were determined in conditioned medium-treated HUVECS with pathway inhibitors as indicated. b Respiratory potential of conditioned medium-treated HUVECs quantified with the Seahorse Metabolic Analyzer. Real-time measurements of oxygen consumption rate (OCR) were obtained basally and then after treatment with oligomycin (ATP synthase inhibitor), carbonyl cyanide p-trifluoromethoxyphenylhydrazone (FCCP, electron transport chain accelerator), and rotenone plus antimycin A (electron transport chain inhibitors). c Representative photomicrographs and quantitative analysis of tube formation assay in conditioned medium-treated HUVECs with and without ERK inhibitor U0126. Scale bar $=100 \mu \mathrm{m}$. d Representative immunoblot images and quantitative analysis in conditioned medium-treated HUVECS with and without ERK inhibitor U0126. e Expression of pro-angiogenesis factors in conditioned medium-treated HUVECS with and without ERK inhibitor U0126. ns, non-significance, ${ }^{*} P<.05,{ }^{* *} P<.01,{ }^{* * *} P<.001$ 


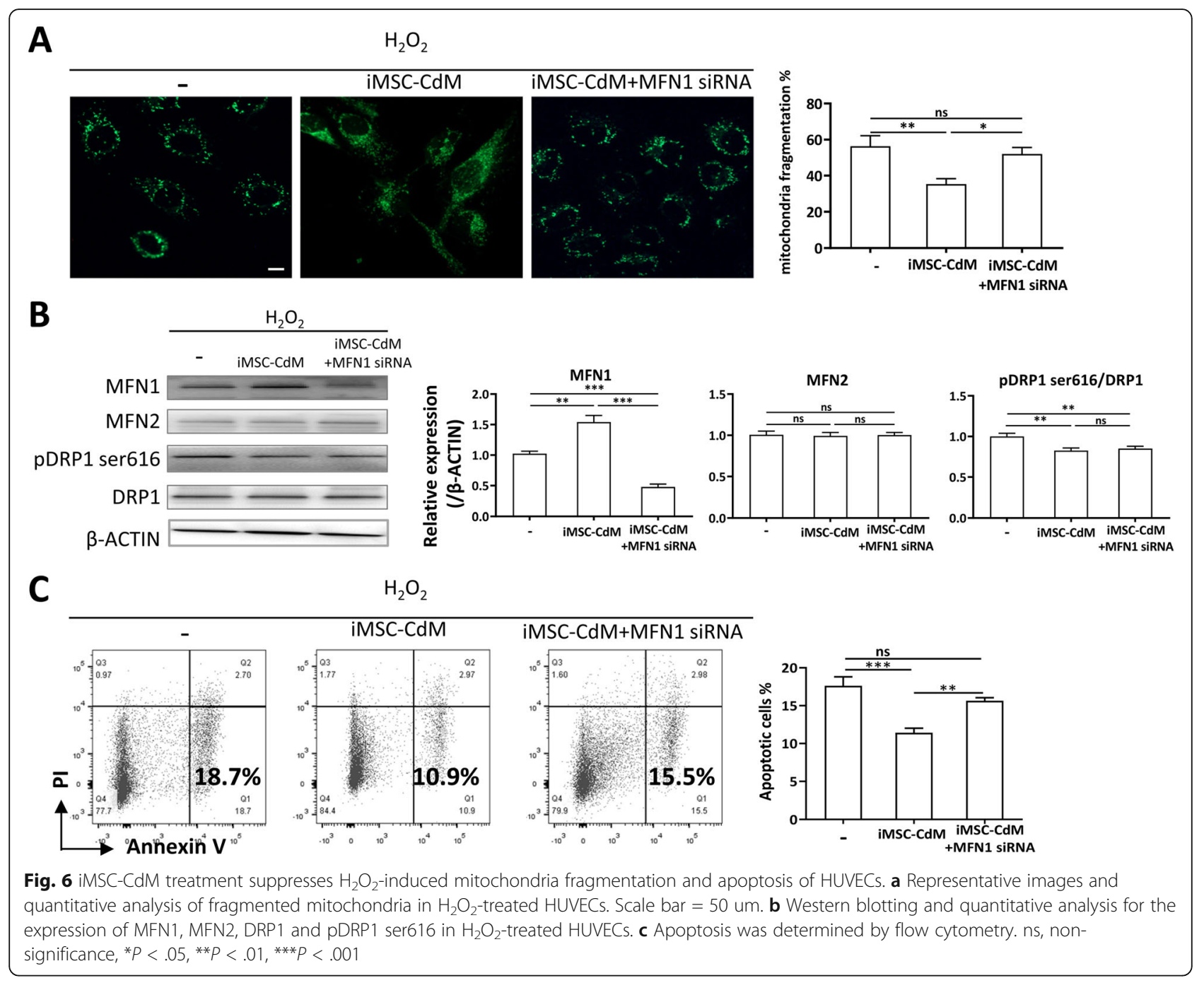

and function. The result showed 76/84 (90.5\%) genes were downregulated in the iMSC-CdM + MFN1 siRNA group compared to the iMSC-CdM group (Supplementary Figure 3), including genes encoding subunits of ATP synthesis, complex I, complex II, complex III (Table 2). Collectively, these observations indicated that iMSC-CdM protected cells from $\mathrm{H}_{2} \mathrm{O}_{2}$-induced injury by regulating mitochondria dynamics.

\section{Discussion}

MSCs have been considered a promising source for cutaneous wound healing. However, accumulating studies showed that MSCs functioned mainly through immune regulation and paracrine effects rather than direct differentiation [25, 26]. Based on the principle of the paracrine mechanism, MSC conditioned medium contains the secretome of the MSCs and is, therefore, a rich source of paracrine factors. MSCs from different sources have similar phenotypic characteristics but differ in secretome profile $[27,28]$. In the current study, we compared the therapeutic effects of iMSC-CdM and uMSC-CdM in treating cutaneous wound healing, and several major findings are as follows: first, iMSC-CdM accelerated wound closure more than uMSC-CdM did (Fig. 2). Histological examinations showed an increase in endothelial marker CD31 and angiogenic factors expression in the peri-wound area in the iMSC-CdM group compared to the uMSC-CdM group (Fig. 3), suggesting the enhanced angiogenesis might involve in iMSC$\mathrm{CdM}$ mediated therapeutics. Following these findings, in vitro studies revealed that iMSC-CdM induced HUVEC proliferation, tube formation, and angiogenic factor expression through activating ERK pathway (Fig. 4e-g; Fig. 5c-e). The excessive reactive oxygen species (ROS) accumulated in the wound is an essential factor in delayed healing. Long-term instability and high concentrations of ROS will eventually lead to insufficient neovascularization, making blood supply and nutritional requirements unable to meet the 
Table 2 List of the genes up- or downregulated (> 2 fold) in the iMSC-CdM + MFN1 siRNA group with respect to iMSC-CdM group

\begin{tabular}{|c|c|c|c|c|}
\hline Position & Symbol & RefSeq number & Description & Fold regulation \\
\hline $\mathrm{A} 01$ & ATP5A1 & NM_004046 & ATP synthase, $\mathrm{H}+$ transporting, mitochondrial F1 complex, alpha subunit 1, cardiac muscle & -2.33 \\
\hline A03 & ATP5C1 & NM_005174 & ATP synthase, H+ transporting, mitochondrial F1 complex, gamma polypeptide 1 & -2.21 \\
\hline A04 & ATP5F1 & NM_001688 & ATP synthase, $\mathrm{H}+$ transporting, mitochondrial Fo complex, subunit B1 & -2.29 \\
\hline A05 & ATP5G1 & NM_005175 & ATP synthase, $\mathrm{H}+$ transporting, mitochondrial Fo complex, subunit C1 (subunit 9) & -2.44 \\
\hline A08 & ATP5H & NM_006356 & ATP synthase, $\mathrm{H}+$ transporting, mitochondrial Fo complex, subunit $\mathrm{d}$ & -2.03 \\
\hline A11 & ATP5J2 & NM_004889 & ATP synthase, $\mathrm{H}+$ transporting, mitochondrial Fo complex, subunit F2 & -2.71 \\
\hline $\mathrm{A} 12$ & ATP5L & NM_006476 & ATP synthase, $\mathrm{H}+$ transporting, mitochondrial Fo complex, subunit $\mathrm{G}$ & -3.01 \\
\hline B02 & COX411 & NM_001861 & Cytochrome c oxidase subunit IV isoform 1 & -2.22 \\
\hline B04 & $\operatorname{COX} 5 B$ & NM_001862 & Cytochrome c oxidase subunit Vb & -2.03 \\
\hline B06 & COX6A2 & NM_005205 & Cytochrome c oxidase subunit Vla polypeptide 2 & 2.32 \\
\hline B08 & $\operatorname{cox} 6 \mathrm{C}$ & NM_004374 & Cytochrome c oxidase subunit Vic & -2.3 \\
\hline $\mathrm{CO} 2$ & NDUFA1 & NM_004541 & NADH dehydrogenase (ubiquinone) 1 alpha subcomplex, 1, 7.5 kDa & -2.09 \\
\hline $\mathrm{CO3}$ & NDUFA10 & NM_004544 & NADH dehydrogenase (ubiquinone) 1 alpha subcomplex, 10, $42 \mathrm{kDa}$ & -2.09 \\
\hline CO4 & NDUFA11 & NM_175614 & NADH dehydrogenase (ubiquinone) 1 alpha subcomplex, 11, $14.7 \mathrm{kDa}$ & -2.14 \\
\hline C06 & NDUFA3 & NM_004542 & NADH dehydrogenase (ubiquinone) 1 alpha subcomplex, 3, $9 \mathrm{kDa}$ & -2.89 \\
\hline C09 & NDUFA6 & NM_002490 & NADH dehydrogenase (ubiquinone) 1 alpha subcomplex, 6, 14 kDa & -3.77 \\
\hline $\mathrm{C} 10$ & NDUFA8 & NM_014222 & NADH dehydrogenase (ubiquinone) 1 alpha subcomplex, 8, $19 \mathrm{kDa}$ & -2.57 \\
\hline C11 & NDUFAB1 & NM_005003 & NADH dehydrogenase (ubiquinone) 1, alpha/beta subcomplex, 1, 8 kDa & -3.36 \\
\hline $\mathrm{C} 12$ & NDUFB10 & NM_004548 & NADH dehydrogenase (ubiquinone) 1 beta subcomplex, 10, $22 \mathrm{kDa}$ & -2.52 \\
\hline D05 & NDUFB6 & NM_182739 & NADH dehydrogenase (ubiquinone) 1 beta subcomplex, 6, $17 \mathrm{kDa}$ & -2.46 \\
\hline D06 & NDUFB7 & NM_004146 & NADH dehydrogenase (ubiquinone) 1 beta subcomplex, 7, $18 \mathrm{kDa}$ & -2.66 \\
\hline D07 & NDUFB8 & NM_005004 & NADH dehydrogenase (ubiquinone) 1 beta subcomplex, 8, $19 \mathrm{kDa}$ & -2.5 \\
\hline D08 & NDUFB9 & NM_005005 & NADH dehydrogenase (ubiquinone) 1 beta subcomplex, 9, $22 \mathrm{kDa}$ & -2.02 \\
\hline D09 & NDUFC1 & NM_002494 & NADH dehydrogenase (ubiquinone) 1, subcomplex unknown, 1, $6 \mathrm{kDa}$ & -2.48 \\
\hline D10 & NDUFC2 & NM_004549 & NADH dehydrogenase (ubiquinone) 1, subcomplex unknown, 2, $14.5 \mathrm{kDa}$ & -24.21 \\
\hline D12 & NDUFS2 & NM_004550 & NADH dehydrogenase (ubiquinone) Fe-S protein 2, $49 \mathrm{kDa}$ (NADH-coenzyme Q reductase) & -2.02 \\
\hline E02 & NDUFS4 & NM_002495 & NADH dehydrogenase (ubiquinone) Fe-S protein 4, 18 kDa (NADH-coenzyme Q reductase) & -3.19 \\
\hline E04 & NDUFS6 & NM_004553 & NADH dehydrogenase (ubiquinone) Fe-S protein 6, $13 \mathrm{kDa}$ (NADH-coenzyme Q reductase) & -2.73 \\
\hline E06 & NDUFS8 & NM_002496 & NADH dehydrogenase (ubiquinone) Fe-S protein 8, $23 \mathrm{kDa}$ (NADH-coenzyme Q reductase) & -2.36 \\
\hline E07 & NDUFV1 & NM_007103 & NADH dehydrogenase (ubiquinone) flavoprotein 1, $51 \mathrm{kDa}$ & -2.46 \\
\hline F01 & SDHC & NM_003001 & Succinate dehydrogenase complex, subunit C, integral membrane protein, $15 \mathrm{kDa}$ & -4.59 \\
\hline F03 & UQCR11 & NM_006830 & Ubiquinol-cytochrome $\mathrm{c}$ reductase, complex III subunit XI & -4.87 \\
\hline F08 & UQCRQ & NM_014402 & Ubiquinol-cytochrome c reductase, complex III subunit VII, 9.5 kDa & -2.77 \\
\hline F09 & ARRDC3 & NM_020801 & Arrestin domain containing 3 & -3.08 \\
\hline F10 & $A S B 1$ & NM_001040445 & Ankyrin repeat and SOCS box containing 1 & -6.03 \\
\hline F11 & CYB561D1 & NM_182580 & Cytochrome b-561 domain containing 1 & -11.48 \\
\hline $\mathrm{F} 12$ & DNAJB & NM_006145 & DnaJ (Hsp40) homolog, subfamily B, member 1 & 2.15 \\
\hline G01 & EDN1 & NM_001955 & Endothelin 1 & 2.14 \\
\hline G04 & HSPA1B & NM_005346 & Heat shock $70 \mathrm{kDa}$ protein 1B & 2.34 \\
\hline G06 & MitoH1 & r4_NC_012920 & Polycistronic_H1_3 & -2.95 \\
\hline
\end{tabular}

Changes in gene expression between groups were evaluated using RT2 Profiler 96-well PCR array plates. Data analysis was done by the $2^{-\triangle \Delta C T}$ method on the manufacturer's web portal (https://geneglobe.qiagen.com/cn/analyze), (QIAGEN, CA, USA) 
needs of wound healing [29]. Since we observed enhanced angiogenesis in the iMSC-CdM treated group in vivo, we examined whether iMSC-CdM exerted a protective role in oxidative stress-induced cell injury in vitro. $\mathrm{H}_{2} \mathrm{O}_{2}$ was applied as the inducer of ROS. The result demonstrated that iMSC-CdM prevented HUVECs from apoptosis, reduced cellular ROS and mitochondrial ROS levels induced by $\mathrm{H}_{2} \mathrm{O}_{2}$ (Fig. 4ac). The $\mathrm{H}_{2} \mathrm{O}_{2}$ induced mPTP openings may release a ROS burst leading to the destruction of mitochondria or even the cell. The iMSC-CdM treatment prevented HUVECs from $\mathrm{H}_{2} \mathrm{O}_{2}$ induced mPTP opening (Fig. 4d). Mitochondria are an important source of ROS generation and energy production. In Fig. $5 \mathrm{a}$, b, we measured the effects of iMSC-CdM on HUVECs energy metabolism and showed iMSC-CdM increased the basal respiratory capacity and ATP production. Collectively, these data suggested the role of iMSC$\mathrm{CdM}$ on mitochondria function. As highly dynamic organelles, mitochondria undergo coordinated cycles of fission and fusion, referred to as "mitochondrial dynamics," to maintain their shape, distribution, and size [30]. We thus determined whether iMSC-CdM regulated mitochondrial dynamics in HUVECs. The data showed iMSC-CdM reduced $\mathrm{H}_{2} \mathrm{O}_{2}$-induced mitochondrial fragmentation and apoptosis, accompanied by decreased pDRP1-ser616 and increased MFN1 expression. These data revealed that iMSC-CdM mediated cytoprotective effects by regulating mitochondria dynamics. In summary, the in vivo data suggested iMSC-CdM accelerated wound healing via enhanced angiogenesis, the in vitro data supported the role of iMSC-CdM in pro-angiogenesis, and the regulation of mitochondria dynamic balance might play a role in iMSC-CdM mediated cytoprotection under oxidative stress.

MSC-based therapy is promising in tissue regeneration and wound repair because it can enhance reepithelialization, increase angiogenesis, modulate inflammation, and regulate extracellular matrix remodeling [31]. In previous studies on wound healing, MSCs have been injected locally into or around the wound area. However, the diseased microenvironment is not conducive to MSC survival and retention, which limits treatment effects. Alternatively, applying the MSC-conditioned medium-which contains the paracrine factors-directly to the wound has considerable therapeutic value $[32,33]$. Hu et al. reported that injections of conditioned medium from bone-marrowderived MSCs attenuated hypertrophic scar formation and accelerated re-epithelization in a rabbit ear wound model [34]. Sun et al. showed that Wharton's jelly-derived MSC-CdM significantly accelerated wound closure and enhanced wound healing quality versus a positive control (epidermal growth factor) [12]. However, MSCs derived from different sources have different secretory profiles, which might affect their potential therapeutic values. More research is needed to determine the optimal source of MSCs for wound treatment.

iMSCs are emerging as a new source of MSCs due to their potential to overcome several limitations of adult MSCs. First, during in vitro expansion, MSCs derived from adult tissues gradually acquire a senescent phenotype, while iMSCs display greater expansion capacity with differentiation potential and normal karyotypes for over 120 doublings [6]. Second, donor age is a decisive factor for the length of the cultivation lifespan and quality of adult MSCs, which hobbles the application of MSCs isolated from aged donors [4]. Alternatively, irrespective of donor age or cell type, iMSCs acquire a rejuvenation-associated gene signature during the reprogramming process [35]. The telomerase activity of iMSCs is 10 times greater than that of adult bone marrow MSCs [6]. Third, adult MSCs are complex cell populations that exhibit intraheterogeneity, which might be one of the explanations for the inconsistent clinical efficacy. iMSCs may represent a more homogenous population as they grew from a single induced pluripotent stem cell clone. More importantly, studies have highlighted the superiority of iMSCs over the other sources of MSCs for the same applications. Lian et al. demonstrated that iMSCs functioned better than native bone marrow MSCs to recover blood perfusion in a mouse hindlimb ischemia model [6]. Liang et al. reported that iMSCs were more effective than bone marrow MSCs in restoring cigarette smoke-induced cardiac dysfunction via inhibiting the NF-kB pathway [9]. iMSC-CdM reduced cigarette smoke medium-induced mitochondrial ROS in airway smooth muscle cells [10]. Soontararak et al. reported that iMSCs were equivalent to, or in some cases, superior to conventional adipose-derived MSCs in treating inflammatory bowel disease in terms of alleviating clinical signs of colitis and stimulating intestinal healing [36]. These data point to iMSCs as a sustainable source of MSCs for clinical applications. In line with these results, our data showed that $\mathrm{iMSC}-\mathrm{CdM}$ was superior to uMSC-CdM to treat wounds. With the same seeded cell number, iMSC-CdM yielded a higher protein concentration, which contained a higher level of angiogenesis factors, including angiogenin, VEGFA, PDGF-BB, HGF and FGF2, than uMSC-CdM. At the cellular level, iMSC$\mathrm{CdM}$ induced HUVEC proliferation and tube formation, increased cellular ATP levels and energy metabolism more than uMSC-CdM did. At the organism level, iMSC-CdM treatment significantly reduced inflammatory cytokine expression and enhanced angiogenesis at the peri-wound area. Excessive production of ROS 
causes oxidative damage, which is the leading cause of non-healing chronic wounds [37]. We measured the effects of conditioned medium on HUVECs under $\mathrm{H}_{2} \mathrm{O}_{2}$ stress. The protective effects of iMSC-CdM on HUVECs were more evident than uMSC-CdM in $\mathrm{H}_{2} \mathrm{O}_{2}$ induced apoptosis. Excessive or inappropriately localized ROS induced cell death, apoptosis and senescence [38]. The main sources of cellular ROS are mitochondria and NADPH oxidases [39]. Cellular ROS and mitochondrial ROS were measured in $\mathrm{H}_{2} \mathrm{O}_{2}$-treated HUVECs. iMSC$\mathrm{CdM}$ showed superior capacity in reducing cellular ROS and equivalent capacity in reducing mitochondrial ROS compared to uMSC-CdM. The mPTP plays a crucial physiological role in maintaining healthy mitochondrial homeostasis. At higher ROS levels, longer MPTP openings may release excessive ROS, leading to the destruction of mitochondria or even the cell [40]. The data showed that iMSC-CdM prevented $\mathrm{H}_{2} \mathrm{O}_{2}$-induced $\mathrm{mPTP}$ opening more effectively than uMSC-CdM did. Mechanistically, iMSC-CdM induced angiogenesis and enhanced energy metabolism mainly through activating the ERK pathway. The ERK inhibitor U0126 partially neutralized the effects of iMSC-CdM on HUVECs, including the ATP levels, energy metabolism and expression of angiogenesis factors.

Mitochondria serve as the energy factory of cells; they maintain cellular homeostasis by participating in ATP production, calcium homeostasis, oxidative stress response, and apoptosis [41]. Mitochondria are constantly moving and undergoing morphologic changes controlled by the mitochondrial fusion and fission process. Mitochondria fusion, regulated by MFN1, MFN2, and OPA1 mitochondrial dynamin-like GTPase (OPA1), generates mitochondrial tubule networks. Mitochondria fission, regulated by DRP1 and fission-1 (FIS1), forms smaller individual mitochondria [42]. The imbalance between fusion and fission contributes to endothelial dysfunction [43]. Under pathological conditions, altered mitochondrial dynamics, characterized by increased fission and decreased fusion, lead to accumulation of small dysfunctional mitochondria, loss of mitochondrial networks and increased mitochondrial ROS [43, 44]. DRP1 knockdown attenuates cellular ROS and mitochondria ROS production in high glucose-treated HUVECs [45]. Our data showed HUVECs exhibited a small and round mitochondrion under $\mathrm{H}_{2} \mathrm{O}_{2}$ stimulation. The iMSC-CdM treatment mitigated mitochondria fragmentation and apoptosis in $\mathrm{H}_{2} \mathrm{O}_{2}$ treated HUVECs, accompanied by decreased pDRP1-ser616 and increased MFN1 expression, and these effects were abolished in part by MFN1siRNA, suggesting iMSC-CdM mediated cytoprotective effects by regulating mitochondria dynamics.

In the current study, the conditioned medium was topically administered to a cotton pad that covered the wound area. In a preliminary investigation, the conditioned medium was injected around the wound area every 3 days after the injury. Unfortunately, this treatment was not beneficial and even seemed to worsen the healing process. Excessive inflammation caused by repeated injection might be one explanation for the observed effects- increased inflammatory cytokine IFNy was detected in the peripheral lymph nodes, and excessive immune cell infiltration was found in the periwound area (data not shown). In light of the beneficial effects of the conditioned medium in vitro, we modified the delivery method according to previously published protocols [12]. In Sun et al.'s study, MSC-conditioned medium-hydrogel was pipetted onto a radiation wound in rats every 2 days [12]. Here, the protocol was modified by "masking" the wound with a cotton pad and then pipetting conditioned medium onto the pad. This method allowed well-proportioned distribution of the conditioned medium and made it possible to show that conditioned medium was beneficial in accelerating wound healing. This highlights the importance of the delivery method in MSC-based cell-free therapy in treating various diseases. However, the detailed mechanisms of the healing process warrant further investigation.

This study has limitations. Firstly, we showed the protein concentration of iMSC-CdM was much higher than uMSC-CdM based on equivalent cell numbers. The higher concentration of pro-angiogenic factors angiogenin, VEGFA, FGF2, PDGF-BB, and HGF in iMSC$\mathrm{CdM}$ might partially explain the superior proangiogenesis effects as compared to uMSC-CdM. These data suggested iMSC had a more active secretion function compared to uMSC. However, the comparison of the contents in iMSC-CdM and uMSC-CdM, as well as the role of the critical components in iMSC-CdM mediated skin regeneration, needs to be determined by high-performance liquid chromatography-electrospray tandem mass spectrometry (HPLC-MS) and gain and loss-of-function approaches in future studies. Secondly, the current study supports a pro-angiogenic role of iMSC-CdM on endothelial cells during wound healing. The effects of iMSC-CdM on fibroblasts and keratinocytes and whether they contribute to the healing process need further investigation. Third, excessive production of ROS or impaired ROS detoxification causes oxidative damage, which is the leading cause of non-healing chronic wounds. The $\mathrm{H}_{2} \mathrm{O}_{2}$-induced $\mathrm{ROS}$ overproduction was applied to establish an oxidative stress-induced cell injury in the current study. However, the in vitro model using $\mathrm{H}_{2} \mathrm{O}_{2}$ only partially mimicked the diseased microenvironment at a certain stage. Application of other stressors, such as hypoxia, can be helpful for a more comprehensive understanding of how iMSC-CdM affects the wound healing process at different stages. 


\section{Conclusion}

Collectively, the data showed that iMSC-CdM has more significant therapeutic potential than uMSC-CdM in treating cutaneous skin injury in mice. As a novel cellfree therapeutic approach, iMSC-CdM avoids the ethical issues of cell transplantation and the risk of tumorigenesis. These data support the use of iMSC-CdM for enhancing wound healing or other tissue regeneration.

\section{Abbreviations}

MSC: Mesenchymal stem cell; uMSC: Umbilical cord MSCs; uMSC-

CdM: Conditioned medium from UMSCs; iMSC: Induced pluripotent stem cells derived MSCs; iMSC-CdM: Conditioned medium from iMSCs; HUVEC: Human umbilical vein endothelial cell; CD: Cluster of differentiation; EGF: Epidermal growth factor; ANG-2: Angiopoietin 2; PIGF: Placenta growth factor; VEGFA: Vascular endothelial growth factor A; HGF: Hepatocyte growth factor; FGF2: Fibroblast growth factor 2; IL6: Interleukin 6; MCP1: Monocyte chemoattractant protein-1; TNFa: Tumor necrosis factor-alpha; IFNY: Interferon Y; IL10: Interleukin 10; IL12p70: Interleukin 12p70; GAPD H: Glyceraldehyde 3-phosphate dehydrogenase; ROS: Reactive oxygen species; mPTP: Mitochondrial permeability transition pore; ATP: Adenosine triphosphate; ERK1/2: Extracellular signal-regulated kinase1/2; pERK1/ 2: Phosphorylated ERK1/2; MFN1: Mitofusin1; MFN2: Mitofusin2; DRP1: Dynamin-related protein1; pDRP1 ser616: Phosphorylated DRP1 ser616; FIS1: Fission-1; FBS: Fetal bovine serum; DAPI: 2-(4-Amidinophenyl)-6indolecarbamidine dihydrochloride

\section{Supplementary Information}

The online version contains supplementary material available at https://doi. org/10.1186/s13287-021-02366-x.

Additional file 1.

\section{Acknowledgements}

The authors thank Dr. Ying Lu and Dr. Xiuhua Li (Clinical Translational Medical Research Center, Shanghai East Hospital, Shanghai) for excellent technical assistance in flow cytometry analysis.

\section{Authors' contributions}

XT Liang: conception and design, experiment performance, data analysis, manuscript writing, financial support; F Lin: experiment performance, data analysis; Y Ding: experiment performance, manuscript writing; YL Zhang: data analysis, manuscript writing; MM Li, L Wei, XX Ma: experiment performance (mouse wound healing model); XH Zhou: provision of study material, data analysis; QS Meng: data analysis; HM Fan: conception and design, provision of study material, financial support, manuscript writing; ZM Liu: conception and design, financial support, manuscript writing. The authors read and approved the final manuscript.

\section{Funding}

This research was supported in part by the National Natural Science Grant of China $(81500207 ; 81700259$, 81770094), the Fundamental Research Funds for the Central Universities (22120180103), the Science and Technology Commission of Shanghai Municipality (17431906600), the National Key Research and Development Program of China (2017YFA0105600), Major Program of Development Fund for Shanghai Zhangjiang National Innovation Demonstration Zone (ZJ2018-ZD-004) and Peak Disciplines (Type IV) of Institutions of Higher Learning in Shanghai, the Three-Year Plan on Promoting TCM development (ZY[2018-2020]-FWTX-2007), and The Key Discipline of the Health Industry Project of Pudong Health Bureau of Shanghai (PWZxk2017-01)

\section{Availability of data and materials}

The data that support the findings of this study are available on request from the corresponding author.

\section{Declarations}

\section{Ethics approval and consent to participate}

All experimental protocols used in this work were conducted following relevant guidelines and regulations of Tongji University and were approved by the Ethics Committee of the Tongji University for Laboratory Animal Medicine (approval number TJLAC-019-141).

\section{Consent for publication}

Not applicable.

\section{Competing interests}

The authors declare no conflicts of interest.

\section{Author details}

${ }^{1}$ Institute for Regenerative Medicine, Shanghai East Hospital, School of Life Sciences and Technology, Tongji University, Shanghai, People's Republic of China. ${ }^{2}$ Research Center for Translational Medicine, Shanghai East Hospital, School of Medicine, Tongji University, Shanghai, People's Republic of China. ${ }^{3}$ Laboratory of Arrhythmias, Ministry of Education of China, Shanghai East Hospital, Tongji University, Shanghai, People's Republic of China. ${ }^{4}$ Department of Organ Transplantation, Changzheng Hospital, Second Military Medical University, Shanghai, People's Republic of China. ${ }^{5}$ Department of Emergency, Guangdong General Hospital, Guangdong Academy of Medical Science, Guangzhou, People's Republic of China. ${ }^{6}$ Department of Cardiovascular Surgery, Shanghai East Hospital, Tongji University, Shanghai, People's Republic of China.

Received: 17 January 2021 Accepted: 2 May 2021

Published online: 20 May 2021

\section{References}

1. Gurtner GC, Werner S, Barrandon Y, Longaker MT. Wound repair and regeneration. Nature. 2008;453(7193):314-21. https://doi.org/10.1038/na ture07039.

2. Hsieh JY, Wang HW, Chang SJ, Liao KH, Lee IH, Lin WS, et al. Mesenchymal stem cells from human umbilical cord express preferentially secreted factors related to neuroprotection, neurogenesis, and angiogenesis. PLoS One. 2013;8(8):e72604. https://doi.org/10.1371/journal.pone.0072604.

3. Kim SW, Zhang HZ, Guo L, Kim JM, Kim MH. Amniotic mesenchymal stem cells enhance wound healing in diabetic NOD/SCID mice through high angiogenic and engraftment capabilities. PLoS One. 2012;7(7):e41105. https://doi.org/10.1371/journal.pone.0041105.

4. Liu J, Ding Y, Liu Z, Liang X. Senescence in mesenchymal stem cells: functional alterations, molecular mechanisms, and rejuvenation strategies. Front Cell Dev Biol. 2020:8:258. https://doi.org/10.3389/fcell.2020.00258.

5. Spitzhorn LS, Megges M, Wruck W, Rahman MS, Otte J, Degistirici Ö, et al. Human iPSC-derived MSCs (iMSCs) from aged individuals acquire a rejuvenation signature. Stem Cell Res Ther. 2019;10(1):100. https://doi.org/1 0.1186/s13287-019-1209-X

6. Lian Q, Zhang Y, Zhang J, Zhang HK, Wu X, Zhang Y, et al. Functional mesenchymal stem cells derived from human induced pluripotent stem cells attenuate limb ischemia in mice. Circulation. 2010;121(9):1113-23. https://doi.org/10.1161/CIRCULATIONAHA.109.898312.

7. Hynes K, Menicanin D, Han J, Marino V, Mrozik K, Gronthos S, et al. Mesenchymal stem cells from iPS cells facilitate periodontal regeneration. J Dent Res. 2013:92(9):833-9. https://doi.org/10.1177/0022034513498258.

8. Moslem M, Valojerdi MR, Pournasr B, Muhammadnejad A, Baharvand H. Therapeutic potential of human induced pluripotent stem cell-derived mesenchymal stem cells in mice with lethal fulminant hepatic failure. Cell Transplant. 2013;22(10):1785-99. https://doi.org/10.3727/096368912X662462.

9. $\quad$ Liang Y, Li X, Zhang Y, Yeung SC, Zhen Z, Ip MSM, et al. Induced pluripotent stem cells-derived mesenchymal stem cells attenuate cigarette smoke-induced cardiac remodeling and dysfunction. Front Pharmacol. 2017; 8:501. https://doi.org/10.3389/fphar.2017.00501.

10. Li X, Michaeloudes C, Zhang Y, Wiegman CH, Adcock IM, Lian Q, et al. Mesenchymal stem cells alleviate oxidative stress-induced mitochondria dysfunction in the airways. J Allergy Clin Immunol. 2018;141(5):1634-45 e1635. https://doi.org/10.1016/j.jaci.2017.08.017. 
11. Chen JS, Wong WW, Gurtner GC. Therapeutic potential of bone marrowderived mesenchymal stem cells for cutaneous wound healing. Front Immunol. 2012;3:192

12. Sun J, Zhang Y, Song $X$, Zhu J, Zhu Q. The healing effects of conditioned medium derived from mesenchymal stem cells on radiation-induced skin wounds in rats. Cell Transplant. 2019;28(1):105-15. https://doi.org/10.1177/ 0963689718807410.

13. Bussche L, Harman RM, Syracuse BA, Plante EL, Lu YC, Curtis TM, et al. Microencapsulated equine mesenchymal stromal cells promote cutaneous wound healing in vitro. Stem Cell Res Ther. 2015;6(1):66. https://doi.org/1 0.1186/s13287-015-0037-x.

14. Veith AP, Henderson K, Spencer A, Sligar AD, Baker AB. Therapeutic strategies for enhancing angiogenesis in wound healing. Adv Drug Deliv Reviews. 2019;146:97-125. https://doi.org/10.1016/j.addr.2018.09.010.

15. Schiffmann LM, Werthenbach JP, Heintges-Kleinhofer F, Seeger JM, Fritsch M, Günther SD, et al. Mitochondrial respiration controls neoangiogenesis during wound healing and tumour growth. Nat Commun. 2020;11(1):3653. https://doi.org/10.1038/s41467-020-17472-2.

16. Diebold LP, Gil HJ, Gao P, Martinez CA, Weinberg SE, Chandel NS. Mitochondrial complex III is necessary for endothelial cell proliferation during angiogenesis. Nature Metab. 2019;1(1):158-71. https://doi.org/10.103 8/s42255-018-0011-x

17. Ponte S, Carvalho L, Gagliardi M, Campos I, Oliveira PJ, Jacinto A. Drp1mediated mitochondrial fission regulates calcium and F-actin dynamics during wound healing. Biol Open. 2020;9(5):bio048629. https://doi.org/1 0.1242/bio.048629.

18. Sun SJ, Lai WH, Jiang Y, Zhen Z, Wei R, Lian Q, et al. Immunomodulation by systemic administration of human-induced pluripotent stem cell-derived mesenchymal stromal cells to enhance the therapeutic efficacy of cellbased therapy for treatment of myocardial infarction. Theranostics. 2021; 11(4):1641-54. https://doi.org/10.7150/thno.46119.

19. Liang $X$, Ding $Y$, Lin F, Zhang $Y$, Zhou $X$, Meng Q, et al. Overexpression of ERBB4 rejuvenates aged mesenchymal stem cells and enhances angiogenesis via PI3K/AKT and MAPK/ERK pathways. FASEB J. 2019;33(3): 4559-70. https://doi.org/10.1096/fj.201801690R.

20. Seluanov A, Vaidya A, Gorbunova V. Establishing primary adult fibroblast cultures from rodents. J Vis Exp. 2010;(44):2033. https://doi.org/10.3791/2033.

21. Jiang D, Chen FX, Zhou H, Lu YY, Tan H, Yu SJ, et al. Bioenergetic crosstalk between mesenchymal stem cells and various ocular cells through the intercellular trafficking of mitochondria. Theranostics. 2020;10(16):7260-72. https://doi.org/10.7150/thno.46332.

22. Deng R, Liu Y, He H, Zhang H, Zhao C, Cui Z, et al. Haemin pre-treatment augments the cardiac protection of mesenchymal stem cells by inhibiting mitochondrial fission and improving survival. J Cell Mol Med. 2020;24(1): 431-40. https://doi.org/10.1111/jcmm.14747.

23. Zhang Y, Guo L, Han S, Chen L, Li C, Zhang Z, et al. Adult mesenchymal stem cell ageing interplays with depressed mitochondrial Ndufs6. Cell Death Dis. 2020;11(12):1075. https://doi.org/10.1038/s41419-020-03289-w.

24. Li P, Gong Z, Shultz LD, Ren G. Mesenchymal stem cells: from regeneration to cancer. Pharmacol Ther. 2019;200:42-54. https://doi.org/10.1016/j.pha rmthera.2019.04.005.

25. Doorn J, Moll G, Le Blanc K, van Blitterswijk C, de Boer J. Therapeutic applications of mesenchymal stromal cells: paracrine effects and potential improvements. Tissue Eng Part B Rev. 2012;18(2):101-15. https://doi.org/10.1 089/ten.teb.2011.0488.

26. Javazon EH, Keswani SG, Badillo AT, et al. Enhanced epithelial gap closure and increased angiogenesis in wounds of diabetic mice treated with adult murine bone marrow stromal progenitor cells. Wound Repair Regen. 2007; 15(3):350-9.

27. Shologu N, Scully M, Laffey JG, O'Toole D. Human mesenchymal stem cell secretome from bone marrow or adipose-derived tissue sources for treatment of hypoxia-induced pulmonary epithelial injury. Int J Mol Sci. 2018;19(10):2996. https://doi.org/10.3390/ijms19102996.

28. Baez-Jurado E, Hidalgo-Lanussa O, Barrera-Bailón B, Sahebkar A, Ashraf GM Echeverria $V$, et al. Secretome of mesenchymal stem cells and its potential protective effects on brain pathologies. Mol Neurobiol. 2019;56(10):6902-27. https://doi.org/10.1007/s12035-019-1570-x.

29. Huang YJ, Nan GX. Oxidative stress-induced angiogenesis. J Clin Neurosci. 2019;63:13-6. https://doi.org/10.1016/j.jocn.2019.02.019.
30. Tilokani L, Nagashima S, Paupe V, Prudent J. Mitochondrial dynamics: overview of molecular mechanisms. Essays Biochem. 2018;62(3):341-60. https://doi.org/10.1042/EBC20170104.

31. Jiang D, Scharffetter-Kochanek K. Mesenchymal stem cells adaptively respond to environmental cues thereby improving granulation tissue formation and wound healing. Front Cell Dev Biol. 2020;8:697. https://doi. org/10.3389/fcell.2020.00697.

32. Zhang $Y$, Niu $X$, Dong $X$, Wang $Y$, Li H. Bioglass enhanced wound healing ability of urine-derived stem cells through promoting paracrine effects between stem cells and recipient cells. J Tissue Eng Regen Med. 2018;12(3): e1609-22. https://doi.org/10.1002/term.2587.

33. Costa MHG, McDevitt TC, Cabral JMS, da Silva CL, Ferreira FC. Tridimensional configurations of human mesenchymal stem/stromal cells to enhance cell paracrine potential towards wound healing processes. J Biotechnol. 2017; 262:28-39. https://doi.org/10.1016/j.jbiotec.2017.09.020.

34. Hu CH, Tseng YW, Chiou CY, Lan KC, Chou CH, Tai CS, et al. Bone marrow concentrate-induced mesenchymal stem cell conditioned medium facilitates wound healing and prevents hypertrophic scar formation in a rabbit ear model. Stem Cell Res Ther. 2019;10(1):275. https://doi.org/10.1186/ s13287-019-1383-x.

35. Frobel J, Hemeda H, Lenz M, Abagnale G, Joussen S, Denecke B, et al. Epigenetic rejuvenation of mesenchymal stromal cells derived from induced pluripotent stem cells. Stem Cell Reports. 2014;3(3):414-22. https://doi.org/1 0.1016/j.stemcr.2014.07.003.

36. Soontararak S, Chow L, Johnson V, Coy J, Wheat W, Regan D, et al. Mesenchymal stem cells (MSC) derived from induced pluripotent stem cells (iPSC) equivalent to adipose-derived MSC in promoting intestinal healing and microbiome normalization in mouse inflammatory bowel disease model. Stem Cells Transl Med. 2018;7(6):456-67. https://doi.org/10.1002/ sctm.17-0305.

37. Cano Sanchez M, Lancel S, Boulanger E, Neviere R. Targeting oxidative stress and mitochondrial dysfunction in the treatment of impaired wound healing: a systematic review. Antioxidants (Basel, Switzerland). 2018;7(8):98. https://doi.org/10.3390/antiox7080098.

38. Ushio-Fukai M, Nakamura Y. Reactive oxygen species and angiogenesis: NADPH oxidase as target for cancer therapy. Cancer Letters. 2008;266(1):3752. https://doi.org/10.1016/j.canlet.2008.02.044.

39. Dan Dunn J, Alvarez LA, Zhang X, Soldati T. Reactive oxygen species and mitochondria: a nexus of cellular homeostasis. Redox Biol. 2015;6:472-85. https://doi.org/10.1016/j.redox.2015.09.005.

40. Zorov DB, Juhaszova M, Sollott SJ. Mitochondrial reactive oxygen species (ROS) and ROS-induced ROS release. Physiol Rev. 2014;94(3):909-50. https:// doi.org/10.1152/physrev.00026.2013.

41. Li A, Gao M, Jiang W, Qin Y, Gong G. Mitochondrial dynamics in adult cardiomyocytes and heart diseases. Front Cell Dev Biol. 2020;8:584800. https://doi.org/10.3389/fcell.2020.584800.

42. Youle RJ, van der Bliek AM. Mitochondrial fission, fusion, and stress. Science. 2012:337(6098):1062-5. https://doi.org/10.1126/science.1219855.

43. Shenouda SM, Widlansky ME, Chen K, Xu G, Holbrook M, Tabit CE, et al. Altered mitochondrial dynamics contributes to endothelial dysfunction in diabetes mellitus. Circulation. 2011;124(4):444-53. https://doi.org/10.1161/ CIRCULATIONAHA.110.014506.

44. Yu T, Robotham JL, Yoon Y. Increased production of reactive oxygen species in hyperglycemic conditions requires dynamic change of mitochondrial morphology. Proc Natl Acad Sci U S A. 2006;103(8):2653-8. https://doi.org/10.1073/pnas.0511154103.

45. Liu H, Xiang H, Zhao S, Sang H, Lv F, Chen R, et al. Vildagliptin improves high glucose-induced endothelial mitochondrial dysfunction via inhibiting mitochondrial fission. J Cell Mol Med. 2019;23(2):798-810. https://doi.org/1 $0.1111 / \mathrm{jcmm} .13975$.

\section{Publisher's Note}

Springer Nature remains neutral with regard to jurisdictional claims in published maps and institutional affiliations. 